\title{
Claim Sizes-Based Perturbed Risk Model with the Dependence Structure
}

\author{
Ying Shen \\ Department of Mathematics, Jining University, Jining, China \\ Email: shenying12345@163.com
}

How to cite this paper: Shen, Y. (2018) Claim Sizes-Based Perturbed Risk Model with the Dependence Structure. Applied Mathematics, 9, 1281-1298.

https://doi.org/10.4236/am.2018.911084

Received: October 17, 2018

Accepted: November 24, 2018

Published: November 27, 2018

Copyright $\odot 2018$ by author and Scientific Research Publishing Inc. This work is licensed under the Creative Commons Attribution International License (CC BY 4.0).

http://creativecommons.org/licenses/by/4.0/

\begin{abstract}
In this paper, we focus on the perturbed risk model with dependent relation and consider the relevance from two aspects. For one side, we use copula function to model the structure of the claim size and interclaim time, and on the other side, we establish the change of premium rat depending on the random thresholds. At last, we obtain the Integro-differential equations and its Laplace transforms of the Gerber-Shiu functions for the new risk model.
\end{abstract}

\section{Keywords}

Perturbed Risk Model, Piecewise Premium, Dependence, The Laplace Transform

\section{Introduction}

Before a hundred years ago, the Lundberg-Cram'er classical model laid the foundation for ruin theory. For its fundamental position, we also call it the compound Poisson risk model, and the surplus process of an insurer is denote by

$$
U(t)=u+c t-S(t)
$$

where $u \geq 0$ denote the initial capital, $c \geq 0$ is the constant premium rate. $S(t)=\sum_{i=1}^{N(t)} X_{i}$ is the aggregate claims until $t$, and $X_{i}$ is the claim in the $i$-th time. $\left\{X_{i}, i \geq 0\right\}$ is a sequence of independent and identically distributed nonnegative variables with a common probability density function $f$ and probability distribution function is $F .\{N(t), t \geq 0\}$ is a Poisson process representing the number of claims in the interval $[0, t]$. We define the ultimate ruin probability by

$$
\psi(u)=\operatorname{Pr}(\tau<\infty \mid U(0)=u)
$$


Because of the rapidly development of the financial and insurance industry, scholars have found that adding perturbations to the original model (1.1) can be better reflect the growth pattern of insurance. They explored many ways, such as linear functions, piecewise functions, levy process, jump-diffusion process, etc. Gerber and Shiu put forward a classical function called the Gerber-Shiu expected discounted penalty functions to study ruin probability better, and they use the Brownian motion to be the perturbation term for the first time. The classical model changes to be

$$
U(t)=u+c t-S(t)+\sigma B(t)
$$

$B(t)$ is a standard Brownian motion and it is independent with the aggregate claims process $S(t)$, and $\sigma>0$ is the diffusion volatility.

The Gerber-Shiu expected discounted penalty function (EDP) is

$$
\phi(u)=E\left[\mathrm{e}^{-\delta \tau} \omega(U(\tau-),|U(\tau)|) I(\tau<\infty) \mid U(0)=u\right], u \geq 0
$$

where $I(\cdot)$ is the indicator function, $\omega\left(x_{1}, x_{2}\right)$ is a no-negative function of the surplus defined on $[0, \infty) \times[0, \infty)$ before ruin $U(\tau-)$ and the deficit at ruin $|U(\tau)|$. Let $\delta \geq 0$ be the force of interest.

Since the compound Poisson risk model perturbed (1.3) and EDP function were proposed, it has received a lot of attention, and the EDP function has been studied fully (including the equation of integro-differential, the Laplace transform, analytic solutions, etc.), see e.g. [1] [2] [3] [4] [5].

At the beginning, researchers consider structure of the claim sizes and the interclaim time in independent for convenient. With the development of research, the independence assumption above does not accord with the actual situation. So many researchers turn to discuss the risk model with various dependent structures, and they built many dependent structures, see e.g. [6]-[15]. Zhang and Yang [13] use the copula function to construct the dependence and obtain better result for the EDP function.

Meanwhile, the research on premium rate is also put forward with the development of insurance industry. Zhou and Cai [15] analyze the dependence structure between the premium rate and the claim size for model (1.3). It doesn't consider the dependence of interclaim time and claim size.

This article is based on the above papers. We study the perturbed risk model as (1.3) and model the dependence structure for two sides. For one side, the interclaim times is dependent with claim sizes by a certain bivariate probability density function, and on the other side, the premiums are depending on claim sizes by the random thresholds $\left\{Q_{i}, i=1,2, \cdots\right\}$. In Section 2, we describe the structure. In Section 3, we analyze the affecting to ruin due to claims or perturbation under the model (1.3) and introduce some ruin measures. We show the integro-differential equations in two situations of Gerber-Shiu function satisfied in Section 4. In Section 5, we get the Laplace transforms for the Section 4.

\section{Analysis of Dependence Structure}

We can use various joint functions to establish dependent structures. In this 
paper, we use the Farlie-Gumbel-Morgenstern (FGM) copula function to describe the dependence structure. We analyze the former perturbed Poisson risk model and establish new dependence structure based on it. Although the FGM function is relatively simple, it can be applied in a variety of environments controlling the size of $\theta$. The FGM copula is shown by

$$
C_{F G M}\left(u_{1}, u_{2}\right)=u_{1} u_{2}+\theta u_{1} u_{2}\left(1-u_{1}\right)\left(1-u_{2}\right), \quad 0 \leq u_{1}, u_{2} \leq 1
$$

where $-1 \leq \theta \leq 1$. We can see that FGM copula also includes the independence case $(\theta=0)$ and allows both negative and positive situations.

We assume $\left\{V_{i}, i \geq 0\right\}$ is the interclaim times and a sequence of exponential random variables $V$, the density function $g(t)=\lambda \mathrm{e}^{-\lambda t} \quad(\lambda>0)$, and the cumulative distribution function $V G(t)=1-\mathrm{e}^{-\lambda t}$. The joint distribution function of $(X, V)$ is

$$
F_{X, V}(x, t)=F(x) G(t)+\theta F(x) G(t)(1-F(x))(1-G(t))
$$

And we can get the joint density function of $(X, V)$ is

$$
f_{X, V}(x, t)=\lambda \mathrm{e}^{-\lambda t} f(x)+\theta\left(2 \lambda \mathrm{e}^{-2 \lambda t}-\lambda \mathrm{e}^{-\lambda t}\right) h(x)
$$

where $g(t)=\lambda \mathrm{e}^{-\lambda t}, h(x)=(1-2 F(x)) f(x)$. We can also get the conditional probability density function, that is

$$
f_{X \mid V=t}(x)=f(x)+\theta\left(2 \mathrm{e}^{-\lambda t}-1\right) h(x)
$$

Then, we analyze the dependence of the premiums and with claim sizes. Let $\left\{Q_{i}, i=1,2, \cdots\right\}$ is a set of independent identical distribution random thresholds and independent with $\left\{X_{i}, i \geq 0\right\}$, the c.d.f. is $L(x)$. If $X_{j}$ is larger than $Q_{j}$, we put the insured on the first group, and we assume the time follows an exponential distribution until the next claim(the p.d.f. is $\left.g_{1}(t)=\lambda_{1} \mathrm{e}^{-\lambda_{1} t}, \lambda_{1}>0\right)$, and the premium at rate $c_{1}(>0)$; if $X_{j}$ is smaller than $Q_{j}$, we put the insured on the second group, the time change exponential parameter (the p.d.f. is $\left.g_{2}(t)=\lambda_{2} \mathrm{e}^{-\lambda_{2} t}, \lambda_{2}>0, \lambda_{1} \neq \lambda_{2}\right)$, and the premium at rate $c_{2}(>0)$. Based on the above assumptions, we denote $C(t)$ the premium growth function and it is a piecewise function.

So we can establish the surplus process of an insurance company in the way blew

$$
\begin{aligned}
U(t) & =u+C(t)-\sum_{i=1}^{N(t)} X_{i}+\sigma B(t) \\
& =u+c_{1} \int_{0}^{t} I_{\{J(s=1)\}} \mathrm{d} s+c_{2} \int_{0}^{t} I_{\{J(s=2)\}} \mathrm{d} s-\sum_{i=1}^{N(t)} X_{i}+\sigma B(t)
\end{aligned}
$$

$J(t)$ is represent two groups of insured persons. To guarantee $U(t)$ has a positive drift, we assume that the model following the next condition

$$
\frac{c_{1}}{\lambda_{1}} P\{X>Q\}+\frac{c_{2}}{\lambda_{2}} P\{X<Q\}-u>0
$$

Following the regulation, the insurance company would charge its premium 
when it is higher than the expected loss amount.

At last, we introduce some functions and formulas that used in this paper.

$$
\varphi_{f}(u)=\int_{u}^{\infty} \omega(u, y-u) f(x) \mathrm{d} x
$$

In this paper, we also use Dickson-Hipp operator $T_{r}, r \geq 0$ and some properties following

$$
\begin{gathered}
T_{r} f(x)=\int_{x}^{\infty} \mathrm{e}^{-r(y-x)} f(y) \mathrm{d} y, \\
T_{r} f(0)=\tilde{f}(r), r \geq 0 . \\
T_{r_{1}} T_{r_{2}} f(x)=T_{r_{2}} T_{r_{1}} f(x)=\frac{T_{r_{1}} f(x)-T_{r_{2}} f(x)}{r_{2}-r_{1}}, r_{1}, r_{2} \geq 0, r_{1} \neq r_{2}, \\
T_{r_{1}} T_{r_{2}} f(0)=T_{r_{2}} T_{r_{1}} f(0)=\frac{\tilde{f}\left(r_{1}\right)-\tilde{f}\left(r_{2}\right)}{r_{2}-r_{1}}, r_{1}, r_{2} \geq 0, r_{1} \neq r_{2} .
\end{gathered}
$$

\section{The Gerber-Shiu Function}

Let $\tau_{i}=\inf \{t \geq 0, U(t)<0\}$ be the ruin time for $U(t)$ for the first time under the zero level, and $\tau_{i}=\infty$ if $U(t) \geq 0$ for all $t \geq 0$.

We analyze the EDP function in two situations in initial surplus $u$

$$
\phi_{i}(u)=E\left[\mathrm{e}^{-\delta \tau_{i}} \omega\left(U\left(\tau_{i}-\right),|U(\tau)|\right) I\left(\tau_{i}<\infty\right) \mid U(0)=u\right], u \geq 0, i=1,2
$$

Because of the perturbation term, we should decompose the EDP function according to two side, that it is whether the ruin is caused by a claim (denote $\phi_{i, w}(u)$ ) or oscillation (denote $\left.\phi_{i, d}(u)\right)$ And there are four cases for the ruin situation:

$$
\begin{gathered}
\phi_{i}(u)=\phi_{i, d}(u)+\phi_{i, w}(u), i=1,2 . \\
\phi_{i, w}(u)=E\left[\mathrm{e}^{-\delta \tau_{i}} \omega\left(U\left(\tau_{i}-\right),|U(\tau)|\right) I\left(\tau_{i}<\infty, U\left(\tau_{i}-\right)<0\right) \mid U(0)=u\right], \\
i=1,2 \\
\phi_{i, d}(u)=E\left[\mathrm{e}^{-\delta \tau_{i}} \omega\left(U\left(\tau_{i}-\right),|U(\tau)|\right) I\left(\tau_{i}<\infty, U\left(\tau_{i}-\right)=0\right) \mid U(0)=u\right] \\
=\omega(0,0) E\left[\mathrm{e}^{-\delta \tau_{i}} I\left(\tau_{i}<\infty, U\left(\tau_{i}-\right)=0\right) \mid U(0)=u\right]
\end{gathered}
$$

In generally, we suppose that $\omega(0,0)=1$. We set a special setting of $\delta=0$ and $\omega=1$ brings $\phi_{i, w}(u)$ and $\phi_{i, d}(u)$ to the ruin probabilities.

\section{Analysis of the Integro-Differential Equations}

In order to discuss the properties of $\phi_{i, w}(u)$ and $\phi_{i, d}(u)$ functions, we usually have to get the Integro-differential equations at first.

Now we use a Brownian motion $W_{i}(t)=-c_{i} t-\sigma B(t), i=1,2$ for an auxiliary function, and the drift is $-c_{i}$ begin with 0 . The variance is $\sigma^{2}$. Let $\bar{W}_{i}(t)=\sup _{0 \leq s \leq t} W_{i}(s)$ is the supremum in interval $[0, t]$. Denoting the first hitting time by $\tau_{i}=\inf \left\{t \geq 0: W_{i}(t)=u\right\}$. Based on the Formula (2.0.1) by Borrodin and Salminen [16], we could get for $\delta \geq 0$, 


$$
E\left[\mathrm{e}^{-\delta \tau_{i}}\right]=\mathrm{e}^{-\eta_{i} u}, \text { where } \eta_{i}=\frac{c_{i}}{\sigma^{2}}+\sqrt{\frac{2 \delta}{\sigma^{2}}+\frac{c_{i}^{2}}{\sigma^{4}}}, \quad i=1,2 .
$$

For $\delta \geq 0$, we define the following measure

$$
\begin{gathered}
P_{i}(u, \mathrm{~d} y, \mathrm{~d} x)=E\left[\mathrm{e}^{-\delta V} I\left(\bar{W}_{i}(V)<u, W_{i}(V) \in \mathrm{d} y, X \in \mathrm{d} x\right)\right], \\
u>0, x>0, y<u, i=1,2 .
\end{gathered}
$$

We denote $e_{q}$ by an exponential random variable and the rate $q>0$. We could first calculate the following measure.

$$
U_{q, i}(u, \mathrm{~d} y)=\operatorname{Pr}\left[\bar{W}_{i}\left(e_{q}\right)<u, W_{i}\left(e_{q}\right) \in \mathrm{d} y\right], u>0, y<u, i=1,2 .
$$

Next, we introduce two Lemmas in applied probability to obtain the above Formula (4.3).

Lemma 1. Assume that $e_{q}$ is independent with $\left\{W_{i}(t)\right\}$. The following random variables $\bar{W}_{i}\left(e_{q}\right), \bar{W}_{i}\left(e_{q}\right)-W_{i}\left(e_{q}\right), i=1,2$ are independent and have exponentially distributed rates

$$
v_{1, i}=\frac{c_{i}}{\sigma^{2}}+\sqrt{\frac{2 q}{\sigma^{2}}+\frac{c_{i}^{2}}{\sigma^{4}}}, v_{2, i}=-\frac{c_{i}}{\sigma^{2}}+\sqrt{\frac{2 q}{\sigma^{2}}+\frac{c_{i}^{2}}{\sigma^{4}}}, i=1,2 .
$$

Respectively, we have for $0 \leq y<u$,

$$
\begin{aligned}
U_{q, i}(u, \mathrm{~d} y) & =\int_{x \in[y, u)} \operatorname{Pr}\left[\overline{W_{i}}\left(e_{q}\right) \in \mathrm{d} x, W_{i}\left(e_{q}\right)-\bar{W}_{i}\left(e_{q}\right)+\bar{W}_{i}\left(e_{q}\right) \in \mathrm{d} y\right] \\
& =\int_{x \in[y, u)} v_{1, i} \mathrm{e}^{-v_{1, i} x} v_{2, i} \mathrm{e}^{-v_{2, i}(x-y)} \mathrm{d} x \mathrm{~d} y \\
& =\frac{v_{1, i} v_{2, i}}{v_{1, i}+v_{2, i}}\left[\mathrm{e}^{-v_{1, i} y}-\mathrm{e}^{-\left(v_{1, i}+v_{2, i}\right) u+v_{2, i} y}\right] \mathrm{d} y
\end{aligned}
$$

We have for $y<0$,

$$
\begin{aligned}
U_{q, i}(u, \mathrm{~d} y) & =\int_{x \in[0, u)} \operatorname{Pr}\left[\overline{W_{i}}\left(e_{q}\right) \in \mathrm{d} x, W_{i}\left(e_{q}\right)-\overline{W_{i}}\left(e_{q}\right)+\bar{W}_{i}\left(e_{q}\right) \in \mathrm{d} y\right] \\
& =\int_{x \in[0, u)} v_{1, i} \mathrm{e}^{-v_{1, i} x} v_{2, i} \mathrm{e}^{-v_{2, i}(x-y)} \mathrm{d} x \mathrm{~d} y \\
& =\frac{v_{1, i} v_{2, i}}{v_{1, i}+v_{2, i}}\left[\mathrm{e}^{-v_{1, i} y}-\mathrm{e}^{-\left(v_{2, i}+v_{2, i}\right) u+v_{2, i} y}\right] \mathrm{d} y
\end{aligned}
$$

For each $u>0, U_{q, i}(u, \mathrm{~d} y)$ is absolutely continuous with respect to the Lebesgue measure.

Lemma 2. The measure $P_{i}(u, \mathrm{~d} y, \mathrm{~d} x)$ has a density in the following

For $0 \leq y<u$,

$$
\begin{aligned}
& p_{i}(u, y, x) \\
& =\frac{\lambda_{i} \eta_{1, i} \eta_{2, i}}{\left(\lambda_{i}+\delta\right)\left(\eta_{1, i}+\eta_{2, i}\right)}\left(\mathrm{e}^{-\eta_{1, i} y}-\mathrm{e}^{-\left(\eta_{1, i}+\eta_{2, i}\right) u+\eta_{2, i} y}\right)(f(x)-\theta h(x)) \\
& +\frac{2 \lambda_{i} \theta \omega_{1, i} \omega_{2, i}}{\left(2 \lambda_{i}+\delta\right)\left(\omega_{1, i}+\omega_{2, i}\right)}\left(\mathrm{e}^{-\omega_{1, i} y}-\mathrm{e}^{-\left(\omega_{1, i}+\omega_{2, i}\right) u+\omega_{2, i} y}\right) h(x)
\end{aligned}
$$

And $y<0$, 


$$
\begin{gathered}
p_{i}(u, y, x) \\
=\frac{\lambda_{i} \eta_{1, i} \eta_{2, i}}{\left(\lambda_{i}+\delta\right)\left(\eta_{1, i}+\eta_{2, i}\right)}\left(\mathrm{e}^{\eta_{2, i} y}-\mathrm{e}^{-\left(\eta_{1, i}+\eta_{2, i}\right) u+\eta_{2, i} y}\right)(f(x)-\theta h(x)) \\
+\frac{2 \lambda_{i} \theta \omega_{1, i} \omega_{2, i}}{\left(2 \lambda_{i}+\delta\right)\left(\omega_{1, i}+\omega_{2, i}\right)}\left(\mathrm{e}^{\omega_{2, i} y}-\mathrm{e}^{-\left(\omega_{1, i}+\omega_{2, i}\right) u+\omega_{2, i} y}\right) h(x) \\
\eta_{1, i}=\frac{c_{i}}{\sigma^{2}}+\sqrt{\frac{2\left(\lambda_{i}+\delta\right)}{\sigma^{2}}+\frac{c_{i}^{2}}{\sigma^{4}}}, \eta_{2, i}=-\frac{c_{i}}{\sigma^{2}}+\sqrt{\frac{2\left(\lambda_{i}+\delta\right)}{\sigma^{2}}+\frac{c_{i}^{2}}{\sigma^{4}}}, i=1,2 . \\
\omega_{1, i}=\frac{c_{i}}{\sigma^{2}}+\sqrt{\frac{2\left(2 \lambda_{i}+\delta\right)}{\sigma^{2}}+\frac{c_{i}^{2}}{\sigma^{4}}}, \omega_{2, i}=-\frac{c_{i}}{\sigma^{2}}+\sqrt{\frac{2\left(2 \lambda_{i}+\delta\right)}{\sigma^{2}}+\frac{c_{i}^{2}}{\sigma^{4}}}, i=1,2 .
\end{gathered}
$$

Proof. Take into account the value of $V$

$$
\begin{aligned}
& P_{i}(u, \mathrm{~d} y, \mathrm{~d} x)=\int_{0}^{\infty} \lambda_{i} \mathrm{e}^{-\left(\lambda_{i}+\delta\right) t} f_{X \mid V=t} \operatorname{Pr}\left[\overline{W_{i}}(t)<u, W_{i}(t) \in \mathrm{d} y\right] \mathrm{d} x \mathrm{~d} t \\
& =\int_{0}^{\infty} \lambda_{i} \mathrm{e}^{-\left(\lambda_{i}+\delta\right) t}(f(x)-\theta h(x)) \operatorname{Pr}\left[\overline{W_{i}}(t)<u, W_{i}(t) \in \mathrm{d} y\right] \mathrm{d} x \mathrm{~d} t \\
& \quad+\int_{0}^{\infty} 2 \lambda_{i} \theta \mathrm{e}^{-\left(2 \lambda_{i}+\delta\right) t} h(x) \operatorname{Pr}\left[\overline{W_{i}}(t)<u, W_{i}(t) \in \mathrm{d} y\right] \mathrm{d} x \mathrm{~d} t \\
& =\frac{\lambda_{i}}{\lambda_{i}+\delta}(f(x)-\theta h(x)) U_{\lambda_{i}+\delta, i}(u, \mathrm{~d} y) \mathrm{d} x+\frac{2 \lambda_{i} \theta}{2 \lambda_{i}+\delta} h(x) U_{2 \lambda_{i}+\delta, i}(u, \mathrm{~d} y) \mathrm{d} x
\end{aligned}
$$

With $U_{\lambda_{i}+\delta, i}(u, \mathrm{~d} y)$ and $U_{2 \lambda_{i}+\delta, i}(u, \mathrm{~d} y)$, we could get the results.

Now, we could obtain the equations of integro-differential for the Gerber-Shiu functions by using the above results.

Theorem 1. $\phi_{1, w}(u), \phi_{2, w}(u)$ satisfies the following integro-differential equation

$$
\begin{aligned}
& \phi_{1, w}(u)= \frac{\lambda_{1} \eta_{1,1} \eta_{2,1}}{\left(\lambda_{1}+\delta\right)\left(\eta_{1,1}+\eta_{2,1}\right)}\left(m_{1,1}(u)+T_{\eta_{2,1}} \gamma_{1}(v)-\mathrm{e}^{-\eta_{1,1} u} \tilde{\gamma}_{1}\left(\eta_{2,1}\right)\right) \\
&+\frac{2 \lambda_{1} \theta \omega_{1,1} \omega_{2,1}}{\left(2 \lambda_{1}+\delta\right)\left(\omega_{1,1}+\omega_{2,1}\right)}\left(m_{1,2}(u)+T_{\omega_{2,1}} \gamma_{2}(v)-\mathrm{e}^{-\omega_{1,1} u} \tilde{\gamma}_{2}\left(\omega_{2,1}\right)\right) \\
& \phi_{2, w}(u)= \frac{\lambda_{2} \eta_{1,2} \eta_{2,2}}{\left(\lambda_{2}+\delta\right)\left(\eta_{1,2}+\eta_{2,2}\right)}\left(m_{2,1}(u)+T_{\eta_{2,2}} \gamma_{1}(v)-\mathrm{e}^{-\eta_{1,2} u} \tilde{\gamma}\left(\eta_{2,2}\right)\right) \\
&+\frac{2 \lambda_{2} \theta \omega_{1,2} \omega_{2,2}}{\left(2 \lambda_{2}+\delta\right)\left(\omega_{1,2}+\omega_{2,2}\right)}\left(m_{2,2}(u)+T_{\omega_{2,2}} \gamma_{2}(v)-\mathrm{e}^{-\omega_{1,2} u} \tilde{\gamma}_{2}\left(\omega_{2,2}\right)\right) \\
& m_{i, 1}(u)=\int_{0}^{u} \mathrm{e}^{-\eta_{1, i}(u-v)} \gamma_{1}(v) \mathrm{d} v, i=1,2, \\
& m_{i, 2}(u)=\int_{0}^{u} \mathrm{e}^{-\omega_{1, i}(u-v)} \gamma_{2}(v) \mathrm{d} v, i=1,2 .
\end{aligned}
$$

Proof. At first, we analyze the time and size for the first claim, and utilize the proofed $p_{i}(u, y, x)$, we have

$$
\begin{aligned}
\phi_{1, w}(u)= & \int_{t \in(0, \infty)} \int_{y \in(-\infty, u)} \lambda_{1} \mathrm{e}^{-\left(\lambda_{1}+\delta\right) t} \operatorname{Pr}\left[\overline{W_{1}}(t)<u, W_{1}(t) \in \mathrm{d} y\right] \\
& \times \int_{x \in(0, u-y)}\left[P\left(x>Q_{1}\right) \phi_{1, w}(u-y-x)+P\left(x<Q_{1}\right) \phi_{2, w}(u-y-x)\right] \\
& \times f_{X, V}(x, t) \mathrm{d} x \mathrm{~d} t+\int_{t \in(0, \infty)} \int_{y \in(-\infty, u)} \lambda_{1} \mathrm{e}^{-\left(\lambda_{1}+\delta\right) t} \operatorname{Pr}\left[\overline{W_{1}}(t)<u, W_{1}(t) \in \mathrm{d} y\right] \\
& \times \int_{x \in(u-y, \infty)} \omega(u-y, x-(u-y)) f_{X, V}(x, t) \mathrm{d} x \mathrm{~d} t
\end{aligned}
$$


By lemma 2, we can get the following form

$$
\begin{aligned}
& \phi_{1, w}(u)=\int_{-\infty}^{u} \int_{0}^{u-y}\left[P\left(x>Q_{1}\right) \phi_{1, w}(u-y-x)+P\left(x<Q_{1}\right) \phi_{2, w}(u-y-x)\right] \\
& \times p_{1}(u, x, y) \mathrm{d} x \mathrm{~d} y+\int_{-\infty}^{u} \int_{u-y}^{\infty} \omega(u-y, x-(u-y)) p_{1}(u, x, y) \mathrm{d} x \mathrm{~d} y
\end{aligned}
$$

And

$$
\begin{aligned}
& \phi_{2, w}(u)=\int_{t \in(0, \infty)} \int_{y \in(-\infty, u)} \lambda_{2} \mathrm{e}^{-\left(\lambda_{2}+\delta\right) t} \operatorname{Pr}\left[\overline{W_{2}}(t)<u, W_{2}(t) \in \mathrm{d} y\right] \\
& \quad \times \int_{x \in(0, u-y)}\left[P\left(x>Q_{1}\right) \phi_{1, w}(u-y-x)+P\left(x<Q_{1}\right) \phi_{2, w}(u-y-x)\right] \\
& \quad \times f_{X, V}(x, t) \mathrm{d} x \mathrm{~d} t+\int_{t \in(0, \infty)} \int_{y \in(-\infty, u)} \lambda_{2} e^{-\left(\lambda_{2}+\delta\right) t} \operatorname{Pr}\left[\overline{W_{2}}(t)<u, W_{2}(t) \in \mathrm{d} y\right] \\
& \quad \times \int_{x \in(u-y, \infty)} \omega(u-y, x-(u-y)) f_{X, V}(x, t) \mathrm{d} x \mathrm{~d} t \\
& =\int_{-\infty}^{u} \int_{0}^{u-y}\left[P\left(x>Q_{1}\right) \phi_{1, w}(u-y-x)+P\left(x<Q_{1}\right) \phi_{2, w}(u-y-x)\right] \\
& \quad \times p_{2}(u, x, y) \mathrm{d} x \mathrm{~d} y+\int_{-\infty}^{u} \int_{u-y}^{\infty} \omega(u-y, x-(u-y)) p_{2}(u, x, y) \mathrm{d} x \mathrm{~d} y
\end{aligned}
$$

Let $L(x)=P\left(x>Q_{1}\right), \bar{L}(x)=1-L(x)$, then

$$
\begin{aligned}
& \phi_{1, w}(u)=\int_{-\infty}^{0} \int_{0}^{u-y}\left[L(x) \phi_{1, w}(u-y-x)+\bar{L}(x) \phi_{2, w}(u-y-x)\right] p_{1}(u, x, y) \mathrm{d} x \mathrm{~d} y \\
& +\int_{0}^{u} \int_{0}^{u-y}\left[L(x) \phi_{1, w}(u-y-x)+\bar{L}(x) \phi_{2, w}(u-y-x)\right] p_{1}(u, x, y) \mathrm{d} x \mathrm{~d} y \\
& +\int_{-\infty}^{0} \int_{u-y}^{\infty} \omega(u-y, x-(u-y)) p_{1}(u, x, y) \mathrm{d} x \mathrm{~d} y \\
& +\int_{0}^{u} \int_{u-y}^{\infty} \omega(u-y, x-(u-y)) p_{1}(u, x, y) \mathrm{d} x \mathrm{~d} y \\
& =\frac{\lambda_{1} \eta_{1,1} \eta_{2,1}}{\left(\lambda_{1}+\delta\right)\left(\eta_{1,1}+\eta_{2,1}\right)}\left\{\int_{0}^{u} \int_{0}^{u-y}\left[L(x) \phi_{1, w}(u-y-x)+\bar{L}(x) \phi_{2, w}(u-y-x)\right]\right. \\
& \quad \times\left(\mathrm{e}^{-\eta_{1,1} y}-\mathrm{e}^{-\left(\eta_{1,1}+\eta_{2,1}\right) u+\eta_{2,1} y}\right)(f(x)-\theta h(x)) \mathrm{d} x \mathrm{~d} y \\
& +\int_{0}^{u} \int_{u-y}^{\infty} \omega(u-y, x-(u-y))\left(\mathrm{e}^{-\eta_{1,1} y}-\mathrm{e}^{-\left(\eta_{1,1}+\eta_{2,1}\right) u+\eta_{2,1} y}\right)(f(x)-\theta h(x)) \mathrm{d} x \mathrm{~d} y \\
& +\int_{-\infty}^{0} \int_{0}^{u-y}\left[L(x) \phi_{1, w}(u-y-x)+\bar{L}(x) \phi_{2, w}(u-y-x)\right] \\
& \quad \times\left(\mathrm{e}^{\eta_{2,1} y}-\mathrm{e}^{-\left(\eta_{1,1}+\eta_{2,1}\right) u+\eta_{2,1} y}\right)(f(x)-\theta h(x)) \mathrm{d} x \mathrm{~d} y \\
& \left.+\int_{-\infty}^{0} \int_{u-y}^{\infty} \omega(u-y, x-(u-y))\left(\mathrm{e}^{\eta_{2,1} y}-\mathrm{e}^{-\left(\eta_{1,1}+\eta_{2,1}\right) u+\eta_{2,1}}\right)(f(x)-\theta h(x)) \mathrm{d} x \mathrm{~d} y\right\} \\
& +\frac{2 \lambda_{1} \theta \omega_{1,1} \omega_{2,1}}{\left(2 \lambda_{1}+\delta\right)\left(\omega_{1,1}+\omega_{2,1}\right)}\left\{\int_{0}^{u} \int_{0}^{u-y}\left[L(x) \phi_{1, w}(u-y-x)+\bar{L}(x) \phi_{2, w}(u-y-x)\right]\right. \\
& \times\left(\mathrm{e}^{-\omega_{1,1} y}-\mathrm{e}^{-\left(\omega_{1,1}+\omega_{2,1}\right) u+\omega_{2,1} y}\right) h(x) \mathrm{d} x \mathrm{~d} y \\
& +\int_{0}^{u} \int_{u-y}^{\infty} \omega(u-y, x-(u-y))\left(\mathrm{e}^{-\omega_{1,1} y}-\mathrm{e}^{-\left(\omega_{1,1}+\omega_{2,1}\right) u+\omega_{2,1} y}\right) h(x) \mathrm{d} x \mathrm{~d} y \\
& +\int_{-\infty}^{0} \int_{0}^{u-y}\left[L(x) \phi_{1, w}(u-y-x)+\bar{L}(x) \phi_{2, w}(u-y-x)\right] \\
& \times\left(\mathrm{e}_{2,1, y}^{\omega_{2,1}}-\mathrm{e}^{-\left(\omega_{1,1}+\omega_{2,1}\right) u+\omega_{2,1} y}\right) h(x) \mathrm{d} x \mathrm{~d} y \\
& \left.+\int_{-\infty}^{0} \int_{u-y}^{\infty} \omega(u-y, x-(u-y))\left(\mathrm{e}^{\omega_{2,1} y}-\mathrm{e}^{-\left(\omega_{1,1}+\omega_{2,1}\right) u+\omega_{2,1} y}\right) h(x) \mathrm{d} x \mathrm{~d} y\right\}
\end{aligned}
$$

In the same way, 


$$
\begin{aligned}
& \phi_{2, w}(u) \\
& =\frac{\lambda_{2} \eta_{1,2} \eta_{2,2}}{\left(\lambda_{2}+\delta\right)\left(\eta_{1,2}+\eta_{2,2}\right)}\left\{\int_{0}^{u} \int_{0}^{u-y}\left[L(x) \phi_{1, w}(u-y-x)+\bar{L}(x) \phi_{2, w}(u-y-x)\right]\right. \\
& \times\left(\mathrm{e}^{-\eta_{12} y}-\mathrm{e}^{-\left(\eta_{1,2}+\eta_{2,2}\right) u+\eta_{2,2} y}\right)(f(x)-\theta h(x)) \mathrm{d} x \mathrm{~d} y \\
& +\int_{0}^{u} \int_{u-y}^{\infty} \omega(u-y, x-(u-y))\left(\mathrm{e}^{-\eta_{1,2} y}-\mathrm{e}^{-\left(\eta_{1,2}+\eta_{2,2}\right) u+\eta_{2,2} y}\right)(f(x)-\theta h(x)) \mathrm{d} x \mathrm{~d} y \\
& +\int_{-\infty}^{0} \int_{0}^{u-y}\left[L(x) \phi_{1, w}(u-y-x)+\bar{L}(x) \phi_{2, w}(u-y-x)\right] \\
& \times\left(\mathrm{e}^{-\eta_{2,2} y}-\mathrm{e}^{-\left(\eta_{1,2}+\eta_{2,2}\right) u+\eta_{2,2} y}\right)(f(x)-\theta h(x)) \mathrm{d} x \mathrm{~d} y \\
& \left.+\int_{-\infty}^{0} \int_{u-y}^{\infty} \omega(u-y, x-(u-y))\left(\mathrm{e}^{-\eta_{2,2} y}-\mathrm{e}^{-\left(\eta_{1,2}+\eta_{2,2}\right) u+\eta_{2,2} y}\right)(f(x)-\theta h(x)) \mathrm{d} x \mathrm{~d} y\right\} \\
& +\frac{2 \lambda_{2} \theta \omega_{1,2} \omega_{2,2}}{\left(2 \lambda_{2}+\delta\right)\left(\omega_{1,2}+\omega_{2,2}\right)}\left\{\int _ { 0 } ^ { u } \int _ { 0 } ^ { u - y } \left[L(x) \phi_{1, w}(u-y-x)\right.\right. \\
& \left.+\bar{L}(x) \phi_{2, w}(u-y-x)\right]\left(\mathrm{e}^{-\omega_{1,2} y}-\mathrm{e}^{-\left(\omega_{1,2}+\omega_{2,2}\right) u+\omega_{2,2} y}\right) h(x) \mathrm{d} x \mathrm{~d} y \\
& +\int_{0}^{u} \int_{u-y}^{\infty} \omega(u-y, x-(u-y))\left(\mathrm{e}^{-\omega_{1,2} y}-\mathrm{e}^{-\left(\omega_{1,2}+\omega_{2,2}\right) u+\omega_{2,2} y}\right) h(x) \mathrm{d} x \mathrm{~d} y \\
& +\int_{-\infty}^{0} \int_{0}^{u-y}\left[L(x) \phi_{1, w}(u-y-x)+\bar{L}(x) \phi_{2, w}(u-y-x)\right] \\
& \times\left(\mathrm{e}^{\omega_{2,2} y}-\mathrm{e}^{-\left(\omega_{1,2}+\omega_{2,2}\right) u+\omega_{2,2} y}\right) h(x) \mathrm{d} x \mathrm{~d} y \\
& \left.+\int_{-\infty}^{0} \int_{u-y}^{\infty} \omega(u-y, x-(u-y))\left(\mathrm{e}^{\omega_{2,2} y}-\mathrm{e}^{-\left(\omega_{1,2}+\omega_{2,2}\right) u+\omega_{2,2} y}\right) h(x) \mathrm{d} x \mathrm{~d} y\right\} \\
& +
\end{aligned}
$$

Let

$$
\begin{aligned}
& \chi_{1}(x)=\bar{L}(x)(f(x)-\theta h(x)), \\
& \xi_{1}(x)=L(x)(f(x)-\theta h(x))=(f(x)-\theta h(x))-\chi_{1}(x) \\
& \chi_{2}(x)=\bar{L}(x) h(x), \xi_{2}(x)=L(x) h(x)=h(x)-\chi_{2}(x)
\end{aligned}
$$

so

$$
\begin{aligned}
& \phi_{1, w}(u)=\frac{\lambda_{1} \eta_{1,1} \eta_{2,1}}{\left(\lambda_{1}+\delta\right)\left(\eta_{1,1}+\eta_{2,1}\right)}\left\{\int _ { 0 } ^ { u } \int _ { 0 } ^ { u - y } \left[\xi_{1}(x) \phi_{1, w}(u-y-x)\right.\right. \\
& \left.+\chi_{1}(x) \phi_{2, w}(u-y-x)\right] \times\left(\mathrm{e}^{-\eta_{1,1} y}-\mathrm{e}^{-\left(\eta_{1,1}+\eta_{2,1}\right) u+\eta_{2,1} y}\right) \mathrm{d} x \mathrm{~d} y \\
& +\int_{0}^{u} \int_{u-y}^{\infty} \omega(u-y, x-(u-y))\left(\mathrm{e}^{-\eta_{1,1} y}-\mathrm{e}^{-\left(\eta_{1,1}+\eta_{2,1}\right) u+\eta_{2,1} y}\right)(f(x)-\theta h(x)) \mathrm{d} x \mathrm{~d} y \\
& +\int_{-\infty}^{0} \int_{0}^{u-y}\left[\xi_{1}(x) \phi_{1, w}(u-y-x)+\chi_{1}(x) \phi_{2, w}(u-y-x)\right] \\
& \quad \times\left(\mathrm{e}^{\eta_{2,1} y}-\mathrm{e}^{-\left(\eta_{1,1}+\eta_{2,1}\right) u+\eta_{2,1} y}\right) \mathrm{d} x \mathrm{~d} y+\int_{-\infty}^{0} \int_{u-y}^{\infty} \omega(u-y, x-(u-y)) \\
& \left.\quad \times\left(\mathrm{e}^{\eta_{2,1} y}-\mathrm{e}^{-\left(\eta_{1,1}+\eta_{2,1}\right) u+\eta_{2,1} y}\right)(f(x)-\theta h(x)) \mathrm{d} x \mathrm{~d} y\right\} \\
& +\frac{2 \lambda_{1} \theta \omega_{1,1} \omega_{2,1}}{\left(2 \lambda_{1}+\delta\right)\left(\omega_{1,1}+\omega_{2,1}\right)}\left\{\int _ { 0 } ^ { u } \int _ { 0 } ^ { u - y } \left[\xi_{2}(x) \phi_{1, w}(u-y-x)\right.\right. \\
& \left.+\chi_{2}(x) \phi_{2, w}(u-y-x)\right]\left(\mathrm{e}^{-\omega_{1,1} y}-\mathrm{e}^{-\left(\omega_{1,1}+\omega_{2,1}\right) u+\omega_{2,1} y}\right) \mathrm{d} x \mathrm{~d} y \\
& +\int_{0}^{u} \int_{u-y}^{\infty} \omega(u-y, x-(u-y))\left(\mathrm{e}^{-\omega_{1,1} y}-\mathrm{e}^{-\left(\omega_{1,1}+\omega_{2,1}\right) u+\omega_{2,1} y}\right) h(x) \mathrm{d} x \mathrm{~d} y \\
& +\int_{-\infty}^{0} \int_{0}^{u-y}\left[\xi_{2}(x) \phi_{1, w}(u-y-x)+\chi_{2}(x) \phi_{2, w}(u-y-x)\right]
\end{aligned}
$$




$$
\begin{aligned}
& \times\left(\mathrm{e}^{\omega_{2,1} y}-\mathrm{e}^{-\left(\omega_{1,1}+\omega_{2,1}\right) u+\omega_{2,1} y}\right) \mathrm{d} x \mathrm{~d} y \\
& \left.+\int_{-\infty}^{0} \int_{u-y}^{\infty} \omega(u-y, x-(u-y))\left(\mathrm{e}^{\omega_{2,1} y}-\mathrm{e}^{-\left(\omega_{1,1}+\omega_{2,1}\right) u+\omega_{2,1} y}\right) h(x) \mathrm{d} x \mathrm{~d} y\right\} \\
& =\frac{\lambda_{1} \eta_{1,1} \eta_{2,1}}{\left(\lambda_{1}+\delta\right)\left(\eta_{1,1}+\eta_{2,1}\right)}\left\{\int_{0}^{u}\left(\mathrm{e}^{-\eta_{1,1} y}-\mathrm{e}^{-\left(\eta_{1,1}+\eta_{2,1}\right) u+\eta_{2,1} y}\right)\right. \\
& \times \int_{0}^{u-y}\left(\left(\xi_{1}(x) \phi_{1, w}(u-y-x)+\chi_{1}(x) \phi_{2, w}(u-y-x)\right) \mathrm{d} x+\varphi_{f-\theta h}(u-y)\right) \mathrm{d} y \\
& +\int_{-\infty}^{0}\left(\mathrm{e}^{\eta_{2,1} y}-\mathrm{e}^{-\left(\eta_{1,1}+\eta_{2,1}\right) u+\eta_{2,1} y}\right) \int_{0}^{u-y}\left(\left(\xi_{1}(x) \phi_{1, w}(u-y-x)\right.\right. \\
& \left.\left.\left.+\chi_{1}(x) \phi_{2, w}(u-y-x)\right) \mathrm{d} x+\varphi_{f-\theta h}(u-y)\right) \mathrm{d} y\right\} \\
& +\frac{2 \lambda_{1} \theta \omega_{1,1} \omega_{2,1}}{\left(2 \lambda_{1}+\delta\right)\left(\omega_{1,1}+\omega_{2,1}\right)}\left\{\int_{0}^{u}\left(\mathrm{e}^{-\omega_{1,1} y}-\mathrm{e}^{-\left(\omega_{1,1}+\omega_{2,1}\right) u+\omega_{2,1} y}\right)\right. \\
& \times \int_{0}^{u-y}\left(\left(\xi_{2}(x) \phi_{1, w}(u-y-x)+\chi_{2}(x) \phi_{2, w}(u-y-x)\right) \mathrm{d} x+\phi_{h}(u-y)\right) \mathrm{d} y \\
& +\int_{-\infty}^{0}\left(\mathrm{e}^{\omega_{2,1} y}-\mathrm{e}^{-\left(\omega_{1,1}+\omega_{2,1}\right) u+\omega_{2,1} y}\right) \int_{0}^{u-y}\left(\left(\xi_{2}(x) \phi_{1, w}(u-y-x)\right.\right. \\
& \left.\left.\left.+\chi_{2}(x) \phi_{2, w}(u-y-x)\right) \mathrm{d} x+\varphi_{h}(u-y)\right) \mathrm{d} y\right\}
\end{aligned}
$$

We bring the change of variable $v=u-y$ into the above equation

$$
\begin{aligned}
& \phi_{1, w}(u)=\frac{\lambda_{1} \eta_{1,1} \eta_{2,1}}{\left(\lambda_{1}+\delta\right)\left(\eta_{1,1}+\eta_{2,1}\right)}\left\{\int_{0}^{u}\left(\mathrm{e}^{-\eta_{1,1}(u-v)}-\mathrm{e}^{-\eta_{1,1} u-\eta_{2,1^{v}}}\right)\right. \\
& \quad \times \int_{0}^{v}\left(\left(\xi_{1}(x) \phi_{1, w}(v-x)+\chi_{1}(x) \phi_{2, w}(v-x)\right) \mathrm{d} x+\varphi_{f-\theta h}(v)\right) \mathrm{d} v \\
& \quad+\int_{u}^{\infty}\left(\mathrm{e}^{\eta_{2,1}(u-v)}-\mathrm{e}^{-\eta_{1,1} u-\eta_{2,1^{v}}}\right) \int_{0}^{v}\left(\left(\xi_{1}(x) \phi_{1, w}(v-x)+\chi_{1}(x) \phi_{2, w}(v-x)\right) \mathrm{d} x\right. \\
& \left.\left.\quad+\varphi_{f-\theta h}(v)\right) \mathrm{d} v\right\}+\frac{2 \lambda_{1} \theta \omega_{1,1} \omega_{2,1}}{\left(2 \lambda_{1}+\delta\right)\left(\omega_{1,1}+\omega_{2,1}\right)}\left\{\int_{0}^{u}\left(\mathrm{e}^{-\omega_{1,1}(u-v)}-\mathrm{e}^{-\omega_{1,1} u-\omega_{2,1^{v}}}\right)\right. \\
& \quad \times \int_{0}^{v}\left(\left(\xi_{2}(x) \phi_{1, w}(v-x)+\chi_{2}(x) \phi_{2, w}(v-x)\right) \mathrm{d} x+\varphi_{h}(v)\right) \mathrm{d} v \\
& \quad+\int_{u}^{\infty}\left(\mathrm{e}^{\omega_{2,1}(u-v)}-\mathrm{e}^{-\omega_{1,1} u-\omega_{2,1^{v}}}\right) \int_{0}^{v}\left(\left(\xi_{2}(x) \phi_{1, w}(v-x)\right.\right. \\
& \left.\left.\left.\quad+\chi_{2}(x) \phi_{2, w}(v-x)\right) \mathrm{d} x+\varphi_{h}(v)\right) \mathrm{d} v\right\} \\
& \phi_{2, w}(u)=\frac{\lambda_{2} \eta_{1,2} \eta_{2,2}}{\left(\lambda_{2}+\delta\right)\left(\eta_{1,2}+\eta_{2,2}\right)}\left\{\int_{0}^{u}\left(\mathrm{e}^{-\eta_{1,2}(u-v)}-\mathrm{e}^{-\eta_{1,2} u-\eta_{2,2} v}\right)\right. \\
& \times \int_{0}^{v}\left(\left(\xi_{1}(x) \phi_{1, w}(v-x)+\chi_{1}(x) \phi_{2, w}(v-x)\right) \mathrm{d} x+\varphi_{f-\theta h}(v)\right) \mathrm{d} y \\
& +\int_{u}^{\infty}\left(\mathrm{e}^{-\eta_{2,2}(u-v)}-\mathrm{e}^{-\eta_{1,2} u-\eta_{2,2^{2}}}\right) \int_{0}^{v}\left(\left(\xi_{1}(x) \phi_{1, w}(v-x)+\chi_{1}(x) \phi_{2, w}(v-x)\right) \mathrm{d} x\right. \\
& \left.\left.+\varphi_{f-\theta h}(v)\right) \mathrm{d} v\right\}+\int_{u}^{\infty}\left(\mathrm{e}^{\omega_{2,2}(u-v)}-\mathrm{e}^{-\omega_{1,2} u-\omega_{2,2^{v}}}\right) \\
& \left.\times \int_{0}^{v}\left(\left(\xi_{2}(x) \phi_{1, w}(v-x)+\chi_{2}(x) \phi_{2, w}(v-x)\right) \mathrm{d} x+\varphi_{h}(v)\right) \mathrm{d} v\right\}
\end{aligned}
$$

Let

$$
\begin{gathered}
\gamma_{1}(y)=\int_{0}^{y}\left(\xi_{1}(x) \phi_{1, w}(y-x)+\chi_{1}(x) \phi_{2, w}(y-x)\right) \mathrm{d} x+\varphi_{f-\theta h}(y), \\
\gamma_{2}(y)=\int_{0}^{y}\left(\xi_{2}(x) \phi_{1, w}(y-x)+\chi_{2}(x) \phi_{2, w}(y-x)\right) \mathrm{d} x+\varphi_{h}(y) .
\end{gathered}
$$


We can rewrite the equation

$$
\begin{aligned}
& \phi_{1, w}(u)=\frac{\lambda_{1} \eta_{1,1} \eta_{2,1}}{\left(\lambda_{1}+\delta\right)\left(\eta_{1,1}+\eta_{2,1}\right)}\left\{\int_{0}^{u}\left(\mathrm{e}^{-\eta_{1,1}(u-v)}-\mathrm{e}^{-\eta_{1,1} u-\eta_{2,1} v}\right) \gamma_{1}(v) \mathrm{d} v\right. \\
& \left.+\int_{u}^{\infty}\left(\mathrm{e}^{\eta_{2,1}(u-v)}-\mathrm{e}^{-\eta_{1,1} u-\eta_{2,1^{v}}}\right) \gamma_{1}(v) \mathrm{d} v\right\} \\
& +\frac{2 \lambda_{1} \theta \omega_{1,1} \omega_{2,1}}{\left(2 \lambda_{1}+\delta\right)\left(\omega_{1,1}+\omega_{2,1}\right)}\left\{\int_{0}^{u}\left(\mathrm{e}^{-\omega_{1,1}(u-v)}-\mathrm{e}^{-\omega_{1,1} u-\omega_{2,1^{v}}}\right) \gamma_{2}(v) \mathrm{d} v\right. \\
& \left.+\int_{u}^{\infty}\left(\mathrm{e}^{\omega_{2,1}(u-v)}-\mathrm{e}^{-\omega_{1,1} u-\omega_{2,1}}\right) \gamma_{2}(v) \mathrm{d} v\right\} \\
& =\frac{\lambda_{1} \eta_{1,1} \eta_{2,1}}{\left(\lambda_{1}+\delta\right)\left(\eta_{1,1}+\eta_{2,1}\right)}\left\{\int_{0}^{u} \mathrm{e}^{-\eta_{1,1}(u-v)} \gamma_{1}(v) \mathrm{d} v\right. \\
& \left.+\int_{u}^{\infty} \mathrm{e}^{\eta_{2,1}(u-v)} \gamma_{1}(v) \mathrm{d} v-\int_{0}^{\infty} \mathrm{e}^{-\eta_{1,1} u-\eta_{2,1} v} \gamma_{1}(v) \mathrm{d} v\right\} \\
& +\frac{2 \lambda_{1} \theta \omega_{1,1} \omega_{2,1}}{\left(2 \lambda_{1}+\delta\right)\left(\omega_{1,1}+\omega_{2,1}\right)}\left\{\int_{0}^{u} \mathrm{e}^{-\omega_{1,1}(u-v)} \gamma_{2}(v) \mathrm{d} v\right. \\
& \left.+\int_{u}^{\infty} \mathrm{e}^{\omega_{2,1}(u-v)} \gamma_{2}(v) \mathrm{d} v-\int_{0}^{\infty} \mathrm{e}^{-\omega_{1,1} u-\omega_{2,1} v} \gamma_{2}(v) \mathrm{d} v\right\} \\
& \phi_{2, w}(u)=\frac{\lambda_{2} \eta_{1,2} \eta_{2,2}}{\left(\lambda_{2}+\delta\right)\left(\eta_{1,2}+\eta_{2,2}\right)}\left\{\int_{0}^{u}\left(\mathrm{e}^{-\eta_{1,2}(u-v)}-\mathrm{e}^{-\eta_{1,2} u-\eta_{2,2} v}\right) \gamma_{1}(v) \mathrm{d} v\right. \\
& \left.+\int_{u}^{\infty}\left(\mathrm{e}^{-\eta_{2,2}(u-v)}-\mathrm{e}^{-\eta_{1,2} u-\eta_{2,2} v}\right) \gamma_{1}(v) \mathrm{d} v\right\} \\
& +\frac{2 \lambda_{2} \theta \omega_{1,2} \omega_{2,2}}{\left(2 \lambda_{2}+\delta\right)\left(\omega_{1,2}+\omega_{2,2}\right)}\left\{\int_{0}^{u}\left(\mathrm{e}^{-\omega_{1,2}(u-v)}-\mathrm{e}^{-\omega_{1,2} u-\omega_{2,2} v}\right) \gamma_{1}(v) \mathrm{d} v\right. \\
& \left.+\int_{u}^{\infty}\left(\mathrm{e}^{\omega_{2,2}(u-v)}-\mathrm{e}^{-\omega_{1,2} u-\omega_{2,2}}\right) \gamma_{2}(v) \mathrm{d} v\right\} \\
& =\frac{\lambda_{2} \eta_{1,2} \eta_{2,2}}{\left(\lambda_{2}+\delta\right)\left(\eta_{1,2}+\eta_{2,2}\right)}\left\{\int_{0}^{u} \mathrm{e}^{-\eta_{1,2}(u-v)} \gamma_{1}(v) \mathrm{d} v\right. \\
& \left.+\int_{u}^{\infty} \mathrm{e}^{-\eta_{2,2}(u-v)} \gamma_{1}(v) \mathrm{d} v-\int_{0}^{\infty} \mathrm{e}^{-\eta_{1,2} u-\eta_{2,2^{v}}} \gamma_{1}(v) \mathrm{d} v\right\} \\
& +\frac{2 \lambda_{2} \theta \omega_{1,2} \omega_{2,2}}{\left(2 \lambda_{2}+\delta\right)\left(\omega_{1,2}+\omega_{2,2}\right)}\left\{\int_{0}^{u} \mathrm{e}^{-\omega_{1,2}(u-v)} \gamma_{1}(v) \mathrm{d} v\right. \\
& \left.+\int_{u}^{\infty} \mathrm{e}^{\omega_{2,2}(u-v)} \gamma_{2}(v) \mathrm{d} v-\int_{0}^{\infty} \mathrm{e}^{-\omega_{1,2} u-\omega_{2,2} v} \gamma_{2}(v)\right\}
\end{aligned}
$$

So

$$
\begin{aligned}
\phi_{1, w}(u)= & \frac{\lambda_{1} \eta_{1,1} \eta_{2,1}}{\left(\lambda_{1}+\delta\right)\left(\eta_{1,1}+\eta_{2,1}\right)}\left(m_{1,1}(u)+T_{\eta_{2,1}} \gamma_{1}(v)-\mathrm{e}^{-\eta_{1,1} u} \tilde{\gamma}_{1}\left(\eta_{2,1}\right)\right) \\
& +\frac{2 \lambda_{1} \theta \omega_{1,1} \omega_{2,1}}{\left(2 \lambda_{1}+\delta\right)\left(\omega_{1,1}+\omega_{2,1}\right)}\left(m_{1,2}(u)+T_{\omega_{2,1}} \gamma_{2}(v)-\mathrm{e}^{-\omega_{1,1} u} \tilde{\gamma}_{2}\left(\omega_{2,1}\right)\right) \\
\phi_{2, w}(u)= & \frac{\lambda_{2} \eta_{1,2} \eta_{2,2}}{\left(\lambda_{2}+\delta\right)\left(\eta_{1,2}+\eta_{2,2}\right)}\left(m_{2,1}(u)+T_{\eta_{2,2}} \gamma_{1}(v)-\mathrm{e}^{-\eta_{1,2} u} \tilde{\gamma}_{1}\left(\eta_{2,2}\right)\right) \\
& +\frac{2 \lambda_{2} \theta \omega_{1,2} \omega_{2,2}}{\left(2 \lambda_{2}+\delta\right)\left(\omega_{1,2}+\omega_{2,2}\right)}\left(m_{2,2}(u)+T_{\omega_{2,2}} \gamma_{2}(v)-\mathrm{e}^{-\omega_{1,2} u} \tilde{\gamma}_{2}\left(\omega_{2,2}\right)\right)
\end{aligned}
$$

Theorem 2. $\phi_{1, d}(u), \phi_{2, d}(u)$ satisfies the following integro-differential equation 


$$
\begin{gathered}
\phi_{1, d}(u)=\mathrm{e}^{-\eta_{1,1} u}+\frac{\lambda_{1} \eta_{1,1} \eta_{2,1}}{\left(\lambda_{1}+\delta\right)\left(\eta_{1,1}+\eta_{2,1}\right)}\left[n_{1,1}(u)+T_{\eta_{2,1}} \alpha_{1}(u)-\mathrm{e}^{-\eta_{1,1} u} \tilde{\alpha}_{1}\left(\eta_{2,1}\right)\right] \\
+\frac{2 \lambda_{1} \theta \omega_{1,1} \omega_{2,1}}{\left(2 \lambda_{1}+\delta\right)\left(\omega_{1,1}+\omega_{2,1}\right)}\left[n_{1,2}(u)+T_{\omega_{2,1}} \alpha_{2}(u)-\mathrm{e}^{-\omega_{1,1} u} \tilde{\alpha}_{2}\left(\omega_{2,1}\right)\right] \\
\phi_{2, d}(u)=\mathrm{e}^{-\eta_{1,2} u}+\frac{\lambda_{2} \eta_{1,2} \eta_{2,2}}{\left(\lambda_{2}+\delta\right)\left(\eta_{1,2}+\eta_{2,2}\right)}\left[n_{2,1}(u)+T_{\eta_{2,2}} \alpha_{1}(u)-\mathrm{e}^{-\eta_{1,2} u} \tilde{\alpha}_{1}\left(\eta_{2,2}\right)\right] \\
+\frac{2 \lambda_{2} \theta \omega_{1,2} \omega_{2,2}}{\left(2 \lambda_{2}+\delta\right)\left(\omega_{1,2}+\omega_{2,2}\right)}\left[n_{2,2}(u)+T_{\omega_{2,2}} \alpha_{2}(u)-\mathrm{e}^{-\omega_{1,2} u} \tilde{\alpha}_{2}\left(\omega_{2,2}\right)\right] \\
n_{i, 1}(u)=\int_{0}^{u} \mathrm{e}^{-\eta_{1, i}(u-v)} \alpha_{1}(v) \mathrm{d} v, i=1,2, \quad n_{i, 2}(u)=\int_{0}^{u} \mathrm{e}^{-\omega_{1, i}(u-v)} \alpha_{2}(v) \mathrm{d} v, i=1,2 .
\end{gathered}
$$

Proof. We analyze the first claim size, and according to whether or not oscillation lead to ruin, we have

$$
\begin{aligned}
\phi_{1, d}(u)= & \int_{0}^{\infty} \lambda_{1} \mathrm{e}^{-\left(\lambda_{1}+\delta\right) t} \int_{-\infty}^{u} \operatorname{Pr}\left(\overline{W_{1}}(t)<u, W_{1}(t) \in \mathrm{d} y\right) \int_{0}^{u-y} f_{X, V}(x, t) \\
& \times\left[P\left(x>Q_{1}\right) \phi_{1, d}(u-y-x)+P\left(x>Q_{2}\right) \phi_{2, d}(u-y-x)\right] \mathrm{d} x \mathrm{~d} t \quad(4.21) \\
& +E\left[\mathrm{e}^{-\delta \tau_{1}} I\left(\tau_{1}<v\right)\right] \\
\phi_{2, d}(u)= & \int_{0}^{\infty} \lambda_{2} \mathrm{e}^{-\left(\lambda_{2}+\delta\right) t} \int_{-\infty}^{u} \operatorname{Pr}\left(\overline{W_{2}}(t)<u, W_{2}(t) \in \mathrm{d} y\right) \int_{0}^{u-y} f_{X, V}(x, t) \\
& \times\left[P\left(x>Q_{1}\right) \phi_{1, d}(u-y-x)+P\left(x>Q_{2}\right) \phi_{2, d}(u-y-x)\right] \mathrm{d} x \mathrm{~d} t \quad(4.22) \\
& +E\left[\mathrm{e}^{-\delta \tau_{2}} I\left(\tau_{2}<v\right)\right]
\end{aligned}
$$

Because of the independence between $V$ and $\{W(t)\}$, we can get the expectation for the random variable exponentially distributed $\left(\tau_{i}, i=1,2\right.$ is the first hitting time).

$$
\begin{aligned}
& E\left[\mathrm{e}^{-\delta \tau_{1}} I\left(\tau_{1}<v\right)\right]=E E\left[\mathrm{e}^{-\delta \tau_{1}} I\left(\tau_{1}<v\right) \mid\{W(t)\}\right]=E\left[\mathrm{e}^{-\left(\lambda_{1}+\delta\right) \tau_{1}}\right]=\mathrm{e}^{-\eta_{1,1} u} \\
& E\left[\mathrm{e}^{-\delta \tau_{2}} I\left(\tau_{2}<v\right)\right]=E E\left[\mathrm{e}^{-\delta \tau_{2}} I\left(\tau_{2}<v\right) \mid\{W(t)\}\right]=E\left[\mathrm{e}^{-\left(\lambda_{2}+\delta\right) \tau_{1}}\right]=\mathrm{e}^{-\eta_{1,2} u}
\end{aligned}
$$

Following the proofed $p_{i}(u, y, x)$, we have

$$
\begin{aligned}
& \phi_{1, d}(u)=\mathrm{e}^{-\eta_{1,1} u}+\int_{-\infty}^{u} \int_{0}^{u-y}\left[L(x) \phi_{1, d}(u-y-x)\right. \\
& \left.\quad+\bar{L}(x) \phi_{2, d}(u-y-x)\right] p_{1}(u, y, x) \mathrm{d} x \mathrm{~d} y \\
& =\mathrm{e}^{-\eta_{1,1} u}+\frac{\lambda_{1} \eta_{1,1} \eta_{2,1}}{\left(\lambda_{1}+\delta\right)\left(\eta_{1,1}+\eta_{2,1}\right)}\left[\int _ { 0 } ^ { u } \int _ { 0 } ^ { u - y } \left[L(x) \phi_{1, d}(u-y-x)\right.\right. \\
& \left.\quad+\bar{L}(x) \phi_{2, d}(u-y-x)\right]\left(\mathrm{e}^{-\eta_{1,1} y}-\mathrm{e}^{-\left(\eta_{1,1}+\eta_{2,1}\right) u+\eta_{2,1} y}\right)(f(x)-\theta h(x)) \mathrm{d} x \mathrm{~d} y \\
& \quad+\int_{-\infty}^{0} \int_{0}^{u-y}\left[L(x) \phi_{1, d}(u-y-x)+\bar{L}(x) \phi_{2, d}(u-y-x)\right] \\
& \left.\quad \times\left(\mathrm{e}^{\eta_{2,1} y}-\mathrm{e}^{-\left(\eta_{1,1}+\eta_{2,1}\right) u+\eta_{2,1} y}\right)(f(x)-\theta h(x)) \mathrm{d} x \mathrm{~d} y\right] \\
& +\frac{2 \lambda_{1} \theta \omega_{1,1} \omega_{2,1}}{\left(2 \lambda_{1}+\delta\right)\left(\omega_{1,1}+\omega_{2,1}\right)}\left[\int _ { 0 } ^ { u } \int _ { 0 } ^ { u - y } \left[L(x) \phi_{1, d}(u-y-x)\right.\right. \\
& \left.+\bar{L}(x) \phi_{2, d}(u-y-x)\right]\left(\mathrm{e}^{-\omega_{1,1} y}-\mathrm{e}^{-\left(\omega_{1,1}+\omega_{2,1}\right) u+\omega_{2,1} y}\right) h(x) \mathrm{d} x \mathrm{~d} y \\
& +\int_{-\infty}^{0} \int_{0}^{u-y}\left[L(x) \phi_{1, d}(u-y-x)+\bar{L}(x) \phi_{2, d}(u-y-x)\right] \\
& \left.\times\left(\mathrm{e}^{\omega_{2,1} y}-\mathrm{e}^{-\left(\omega 1+\omega_{2,1}\right) u+\omega_{2,1} y}\right) h(x) \mathrm{d} x \mathrm{~d} y\right]
\end{aligned}
$$




$$
\begin{aligned}
& =\mathrm{e}^{-\eta_{1,1} u}+\frac{\lambda_{1} \eta_{1,1} \eta_{2,1}}{\left(\lambda_{1}+\delta\right)\left(\eta_{1,1}+\eta_{2,1}\right)}\left\{\int_{0}^{u}\left(\mathrm{e}^{-\eta_{1,1} y}-\mathrm{e}^{-\left(\eta_{1,1}+\eta_{2,1}\right) u+\eta_{2,1} y}\right)\right. \\
& \times\left[\int_{0}^{u-y}\left(\xi_{1}(x) \phi_{1, d}(u-y-x)+\chi_{1}(x) \phi_{2, d}(u-y-x)\right) \mathrm{d} x\right] \mathrm{d} y \\
& +\frac{2 \lambda_{1} \theta \omega_{1,1} \omega_{2,1}}{\left(2 \lambda_{1}+\delta\right)\left(\omega_{1,1}+\omega_{2,1}\right)}\left\{\int_{0}^{u}\left(\mathrm{e}^{-\omega_{1,1} y}-\mathrm{e}^{-\left(\omega_{1,1}+\omega_{2,1}\right) u+\omega_{2,1} y}\right)\right. \\
& \times \int_{0}^{u-y}\left(\xi_{1}(x) \phi_{1, d}(u-y-x)+\chi_{1}(x) \phi_{2, d}(u-y-x)\right) \mathrm{d} x \mathrm{~d} y \\
& +\int_{-\infty}^{0}\left(\mathrm{e}^{\omega_{2,1} y}-\mathrm{e}^{-\left(\omega_{1,1}+\omega_{2,1}\right) u+\omega_{2,1} y}\right) \int_{0}^{u-y}\left[\xi_{2} \phi_{1, d}(u-y-x)\right. \\
& \left.\left.+\chi_{2}(x) \phi_{2, d}(u-y-x)\right] \mathrm{d} x \mathrm{~d} y\right\} \\
& \phi_{2, d}(u)=\mathrm{e}^{-\eta_{1,2} u}+\frac{\lambda_{2} \eta_{1,2} \eta_{2,2}}{\left(\lambda_{2}+\delta\right)\left(\eta_{1,2}+\eta_{2,2}\right)}\left\{\int_{0}^{u}\left(\mathrm{e}^{-\eta_{1,2} y}-\mathrm{e}^{-\left(\eta_{1,2}+\eta_{2,2}\right) u+\eta_{2,2} y}\right)\right. \\
& \times\left[\int_{0}^{u-y}\left(\xi_{1}(x) \phi_{1, d}(u-y-x)+\chi_{1}(x) \phi_{2, d}(u-y-x)\right) \mathrm{d} x\right] \mathrm{d} y \\
& +\int_{-\infty}^{0}\left(\mathrm{e}^{\eta_{2,2} y}-\mathrm{e}^{-\left(\eta_{1,2}+\eta_{2,2}\right) u+\eta_{2,2} y}\right) \int_{0}^{u-y}\left(\xi_{1}(x) \phi_{1, d}(u-y-x)\right\} \\
& \left.+\chi_{1}(x) \phi_{2, d}(u-y-x)\right) \mathrm{d} x \mathrm{~d} y+\frac{2 \lambda_{2} \theta \omega_{1,2} \omega_{2,2}}{\left(2 \lambda_{2}+\delta\right)\left(\omega_{1,2}+\omega_{2,2}\right)} \\
& \times\left\{\int _ { 0 } ^ { u } ( \mathrm { e } ^ { - \omega _ { 1 , 2 } y } - \mathrm { e } ^ { - ( \omega _ { 1 , 2 } + \omega _ { 2 , 2 } ) u + \omega _ { 2 , 2 } y } ) \int _ { 0 } ^ { u - y } \left(\xi_{1}(x) \phi_{1, d}(u-y-x)\right.\right. \\
& \left.+\chi_{1}(x) \phi_{2, d}(u-y-x)\right) \mathrm{d} x \mathrm{~d} y+\int_{-\infty}^{0}\left(\mathrm{e}^{\omega_{2,2} y}-\mathrm{e}^{-\left(\omega_{1,2}+\omega_{2,2}\right) u+\omega_{2,2} y}\right) \\
& \left.\times \int_{0}^{u-y}\left[\xi_{2} \phi_{1, d}(u-y-x)+\chi_{2}(x) \phi_{2, d}(u-y-x)\right] \mathrm{d} x \mathrm{~d} y\right\}
\end{aligned}
$$

We bring the change of variable $v=u-y$ into (4.25) and (4.26)

$$
\begin{aligned}
& \phi_{1, d}=\mathrm{e}^{-\eta_{1,1} u}+\frac{\lambda_{1} \eta_{1,1} \eta_{2,1}}{\left(\lambda_{1}+\delta\right)\left(\eta_{1,1}+\eta_{2,1}\right)}\left\{\int_{0}^{u}\left(\mathrm{e}^{-\eta_{1,1}(u-v)}-\mathrm{e}^{-\eta_{1,1} u-\eta_{2,1} v}\right)\right. \\
& \times\left[\int_{0}^{v}\left(\xi_{1}(x) \phi_{1, d}(v-x)+\chi_{1}(x) \phi_{2, d}(v-x)\right) \mathrm{d} x\right] \mathrm{d} v \\
& \left.+\int_{u}^{\infty}\left(\mathrm{e}^{\eta_{2,1}(u-v)}-\mathrm{e}^{-\eta_{1,1} u-\eta_{2,1^{v}}}\right) \int_{0}^{v}\left(\xi_{1}(x) \phi_{1, d}(v-x)+\chi_{1}(x) \phi_{2, d}(v-x)\right) \mathrm{d} x \mathrm{~d} v\right\} \\
& +\frac{2 \lambda_{1} \theta \omega_{1,1} \omega_{2,1}}{\left(2 \lambda_{1}+\delta\right)\left(\omega_{1,1}+\omega_{2,1}\right)}\left\{\int_{0}^{u}\left(\mathrm{e}^{-\omega_{1,1}(u-v)}-\mathrm{e}^{-\omega_{1,1} u-\omega_{2,1} v}\right)\right. \\
& \quad \times \int_{0}^{v}\left(\xi_{2}(x) \phi_{1, d}(v-x)+\chi_{2}(x) \phi_{2, d}(v-x)\right) \mathrm{d} x \mathrm{~d} v \\
& \left.+\int_{u}^{\infty}\left(\mathrm{e}^{\omega_{2,1}(u-v)}-\mathrm{e}^{-\omega_{1,1} u \omega_{2,1} v}\right) \int_{0}^{v}\left[\xi_{2} \phi_{1, d}(v-x)+\chi_{2}(x) \phi_{2, d}(v-x)\right] \mathrm{d} x \mathrm{~d} v\right\} \\
& =\mathrm{e}^{-\eta_{1,1} u}+\frac{\lambda_{1} \eta_{1,1} \eta_{2,1}}{\left(\lambda_{1}+\delta\right)\left(\eta_{1,1}+\eta_{2,1}\right)}\left\{\int_{0}^{u}\left(\mathrm{e}^{-\eta_{1,1}(u-v)}-\mathrm{e}^{-\eta_{1,1} u-\eta_{2,1^{v}}}\right) \alpha_{1}(v) \mathrm{d} v\right. \\
& \left.+\int_{u}^{\infty}\left(\mathrm{e}^{\eta_{2,1^{v}}}-\mathrm{e}^{-\eta_{1,1} u-\eta_{2,1^{v}}}\right) \alpha_{1}(v) \mathrm{d} v\right\}+\frac{2 \lambda_{1} \theta \omega_{1,1} \omega_{2,1}}{\left(2 \lambda_{1}+\delta\right)\left(\omega_{1,1}+\omega_{2,1}\right)} \\
& \quad \times\left\{\int_{0}^{u}\left(\mathrm{e}^{-\omega_{1,1}(u-v)}-\mathrm{e}^{-\omega_{1,1} u-\omega_{2,1} v}\right) \alpha_{2}(v) \mathrm{d} v+\int_{u}^{\infty}\left(\mathrm{e}^{\omega_{2,1}(u-v)}-\mathrm{e}^{-\omega_{1,1} u-\omega_{2,1} v}\right) \alpha_{2}(v) \mathrm{d} v\right\}
\end{aligned}
$$




$$
\begin{aligned}
& =\mathrm{e}^{-\eta_{1,1} u}+\frac{\lambda_{1} \eta_{1,1} \eta_{2,1}}{\left(\lambda_{1}+\delta\right)\left(\eta_{1,1}+\eta_{2,1}\right)}\left\{\int_{0}^{u} \mathrm{e}^{-\eta_{1,1}(u-v)} \alpha_{1}(v) \mathrm{d} v+\int_{u}^{\infty} \mathrm{e}^{\eta_{2,1^{v}}} \alpha_{1}(v) \mathrm{d} v\right. \\
& \left.-\int_{0}^{\infty} \mathrm{e}^{-\eta_{1,1} u-\eta_{2,1^{v}}} \alpha_{1}(v) \mathrm{d} v\right\}+\frac{2 \lambda_{1} \theta \omega_{1,1} \omega_{2,1}}{\left(2 \lambda_{1}+\delta\right)\left(\omega_{1,1}+\omega_{2,1}\right)}\left\{\int_{0}^{u} \mathrm{e}^{-\omega_{1,1}(u-v)} \alpha_{2}(v) \mathrm{d} v\right. \\
& \left.+\int_{u}^{\infty} \mathrm{e}^{\omega_{2,1} y} \alpha_{2}(v) \mathrm{d} v-\int_{0}^{\infty} \mathrm{e}^{-\omega_{1,1} u-\omega_{2,1} v} \alpha_{2}(v) \mathrm{d} v\right\} \\
& =\mathrm{e}^{-\eta_{1,1} u}+\frac{\lambda_{1} \eta_{1,1} \eta_{2,1}}{\left(\lambda_{1}+\delta\right)\left(\eta_{1,1}+\eta_{2,1}\right)}\left[n_{1,1}(u)+T_{\eta_{2,1}} \alpha_{1}(u)-\mathrm{e}^{-\eta_{1,1} u} \tilde{\alpha}_{1}\left(\eta_{2,1}\right)\right] \\
& +\frac{2 \lambda_{1} \theta \omega_{1,1} \omega_{2,1}}{\left(2 \lambda_{1}+\delta\right)\left(\omega_{1,1}+\omega_{2,1}\right)}\left[n_{1,2}(u)+T_{\omega_{2,1}} \alpha_{2}(u)-\mathrm{e}^{-\omega_{1,1} u} \tilde{\alpha}_{2}\left(\omega_{2,1}\right)\right] \\
& \phi_{2, d}(u)=\mathrm{e}^{-\eta_{1,2} u}+\frac{\lambda_{2} \eta_{1,2} \eta_{2,2}}{\left(\lambda_{2}+\delta\right)\left(\eta_{1,2}+\eta_{2,2}\right)}\left\{\int_{0}^{u}\left(\mathrm{e}^{-\eta_{1,2}(u-v)}-\mathrm{e}^{-\eta_{1,2} u-\eta_{2,2^{2}}}\right) \alpha_{1}(v) \mathrm{d} v\right. \\
& \left.+\int_{u}^{\infty}\left(\mathrm{e}^{\eta_{2,2}(u-v)}-\mathrm{e}^{-\eta_{1,2} u-\eta_{2,2} v}\right) \alpha_{1}(v) \mathrm{d} v\right\}+\frac{2 \lambda_{2} \theta \omega_{1,2} \omega_{2,2}}{\left(2 \lambda_{2}+\delta\right)\left(\omega_{1,2}+\omega_{2,2}\right)} \\
& \times\left\{\int_{0}^{u}\left(\mathrm{e}^{-\omega_{1,2}(u-v)}-\mathrm{e}^{-\omega_{1,2} u-\omega_{2,2} v}\right) \alpha_{2}(v) \mathrm{d} v+\int_{u}^{\infty}\left(\mathrm{e}^{\omega_{2,2}(u-v)}-\mathrm{e}^{-\omega_{1,2} u-\omega_{2,2} v}\right) \alpha_{2}(v) \mathrm{d} v\right\} \\
& =\mathrm{e}^{-\eta_{1,2} u}+\frac{\lambda_{2} \eta_{1,2} \eta_{2,2}}{\left(\lambda_{2}+\delta\right)\left(\eta_{1,2}+\eta_{2,2}\right)}\left\{\int_{0}^{u} \mathrm{e}^{-\eta_{1,2}(u-v)} \alpha_{1}(v) \mathrm{d} v+\int_{u}^{\infty} \mathrm{e}^{\eta_{2,2}(u-v)} \alpha_{1}(v) \mathrm{d} v\right. \\
& -\int_{0}^{\infty} \mathrm{e}^{-\eta_{1,2} u-\eta_{2,2} v} \alpha_{1}(v) \mathrm{d} v+\frac{2 \lambda_{2} \theta \omega_{1,2} \omega_{2,2}}{\left(2 \lambda_{2}+\delta\right)\left(\omega_{1,2}+\omega_{2,2}\right)}\left\{\int_{0}^{u} \mathrm{e}^{-\omega_{1,2}(u-v)} \alpha_{2}(v) \mathrm{d} v\right. \\
& \left.+\int_{u}^{\infty} \mathrm{e}^{\omega_{2,2}(u-v)} \alpha_{2}(v) \mathrm{d} v-\int_{0}^{\infty} \mathrm{e}^{-\omega_{1,2} u-\omega_{2,2} v} \alpha_{2}(v) \mathrm{d} v\right\} \\
& =\mathrm{e}^{-\eta_{1,2} u}+\frac{\lambda_{2} \eta_{1,2} \eta_{2,2}}{\left(\lambda_{2}+\delta\right)\left(\eta_{1,2}+\eta_{2,2}\right)}\left[n_{2,1}(u)+T_{\eta_{2,2}} \alpha_{1}(u)-\mathrm{e}^{-\eta_{1,2} u} \tilde{\alpha}_{1}\left(\eta_{2,2}\right)\right] \\
& +\frac{2 \lambda_{2} \theta \omega_{1,2} \omega_{2,2}}{\left(2 \lambda_{2}+\delta\right)\left(\omega_{1,2}+\omega_{2,2}\right)}\left[n_{2,2}(u)+T_{\omega_{2,2}} \alpha_{2}(u)-\mathrm{e}^{-\omega_{1,2} u} \tilde{\alpha}_{2}\left(\omega_{2,2}\right)\right]
\end{aligned}
$$

and

$$
\begin{aligned}
& \alpha_{1}(y)=\int_{0}^{y}\left[\xi_{1} \phi_{1, d}(y-x)+\chi_{1}(x) \phi_{2, d}(y-x)\right] \mathrm{d} x, \\
& \alpha_{2}(y)=\int_{0}^{y}\left[\xi_{2} \phi_{1, d}(y-x)+\chi_{2}(x) \phi_{2, d}(y-x)\right] \mathrm{d} x .
\end{aligned}
$$

\section{Laplace Transforms}

Firstly, we consider the Laplace transforms of $m_{1, i}(u)$

$$
\begin{aligned}
& \tilde{m}_{1,1}(s)=\int_{0}^{\infty} \mathrm{e}^{-s y} \int_{0}^{y} \mathrm{e}^{-\eta_{1,1}(y-v)} \gamma_{1}(v) \mathrm{d} v \mathrm{~d} y=\int_{0}^{\infty} \int_{v}^{\infty} \mathrm{e}^{-s y} \mathrm{e}^{-\eta_{1,1}(y-v)} \gamma_{1}(v) \mathrm{d} v \mathrm{~d} y \\
&=\int_{0}^{\infty} \mathrm{e}^{\eta_{1,1} v} \frac{1}{s+\eta_{1,1}} \mathrm{e}^{-\left(s+\eta_{1,1}\right) v} \gamma_{1}(v) \mathrm{d} v \\
&=\frac{1}{s+\eta_{1,1}} \int_{0}^{\infty} \mathrm{e}^{-s v} \gamma_{1}(v) \mathrm{d} v=\frac{1}{s+\eta_{1,1}} \tilde{\gamma}_{1}(v) \\
& \int_{0}^{\infty} \mathrm{e}^{-s u} \mathrm{e}^{-\eta_{1,1} v} \tilde{\gamma}_{1}\left(\eta_{2,1}\right) \mathrm{d} u=\tilde{\gamma}_{1}\left(\eta_{2,1}\right) \int_{0}^{\infty} \mathrm{e}^{-\left(s+\eta_{1,1}\right) u} \mathrm{~d} u=\frac{1}{s+\eta_{1,1}} \tilde{\gamma}_{1}\left(\eta_{2,1}\right)
\end{aligned}
$$

So

$$
\tilde{m}_{1,2}(s)=\int_{0}^{\infty} \mathrm{e}^{-s y} \int_{0}^{y} \mathrm{e}^{-\omega_{1,1}(y-v)} \gamma_{2}(v) \mathrm{d} v \mathrm{~d} y=\frac{1}{s+\omega_{1,1}} \tilde{\gamma}_{2}(v)
$$




$$
\begin{gathered}
\int_{0}^{\infty} \mathrm{e}^{-s u} \mathrm{e}^{-\omega_{1,1} v} \tilde{\gamma}_{2}\left(\omega_{2,1}\right) \mathrm{d} u=\tilde{\gamma}_{2}\left(\omega_{2,1}\right) \int_{0}^{\infty} \mathrm{e}^{-\left(s+\omega_{1,1}\right) u} \mathrm{~d} u=\frac{1}{s+\omega_{1,1}} \tilde{\gamma}_{2}\left(\omega_{2,1}\right) \\
\tilde{m}_{2,1}(s)=\int_{0}^{\infty} \mathrm{e}^{-s y} \int_{0}^{y} \mathrm{e}^{-\eta_{1,2}(y-v)} \gamma_{1}(v) \mathrm{d} v \mathrm{~d} y=\frac{1}{s+\eta_{1,2}} \tilde{\gamma}_{1}(v) \\
\int_{0}^{\infty} \mathrm{e}^{-s u} \mathrm{e}^{-\eta_{1,2} v} \tilde{\gamma}_{1}\left(\eta_{2,2}\right) \mathrm{d} u=\tilde{\gamma}_{1}\left(\eta_{2,2}\right) \int_{0}^{\infty} \mathrm{e}^{-\left(s+\eta_{1,2}\right) u} \mathrm{~d} u=\frac{1}{s+\eta_{1,2}} \tilde{\gamma}_{1}\left(\eta_{2,2}\right) \\
\tilde{m}_{2,2}(s)=\int_{0}^{\infty} \mathrm{e}^{-s y} \int_{0}^{y} \mathrm{e}^{-\omega_{1,2}(y-v)} \gamma_{2}(v) \mathrm{d} v \mathrm{~d} y=\frac{1}{s+\omega_{1,2}} \tilde{\gamma}_{2}(v) \\
\int_{0}^{\infty} \mathrm{e}^{-s u} \mathrm{e}^{-\omega_{1,2} v} \tilde{\gamma}_{2}\left(\omega_{2,2}\right) \mathrm{d} u=\tilde{\gamma}_{2}\left(\omega_{2,2}\right) \int_{0}^{\infty} \mathrm{e}^{-\left(s+\omega_{1,2}\right) u} \mathrm{~d} u=\frac{1}{s+\omega_{1,2}} \tilde{\gamma}_{2}\left(\omega_{2,2}\right)
\end{gathered}
$$

And then we consider the Laplace transport

$$
\begin{aligned}
& \tilde{\phi}_{1, w}(s)=\frac{\lambda_{1} \eta_{1,1} \eta_{2,1}}{\left(\lambda_{1}+\delta\right)\left(\eta_{1,1}+\eta_{2,1}\right)}\left(\tilde{m}_{1,1}(s)+T_{s} T_{\eta_{2,1}} \gamma_{1}(0)-\frac{1}{s+\eta_{1,1}} \tilde{\gamma}_{1}\left(\eta_{2,1}\right)\right) \\
& +\frac{2 \lambda_{1} \theta \omega_{1,1} \omega_{2,1}}{\left(2 \lambda_{1}+\delta\right)\left(\omega_{1,1}+\omega_{2,1}\right)}\left(\tilde{m}_{1,2}(s)+T_{s} T_{\omega_{2,1}} \gamma_{2}(0)-\frac{1}{s+\omega_{1,1}} \tilde{\gamma}_{2}\left(\omega_{2,1}\right)\right) \\
& =\frac{\lambda_{1} \eta_{1,1} \eta_{2,1}}{\left(\lambda_{1}+\delta\right)\left(\eta_{1,1}+\eta_{2,1}\right)}\left(\frac{\tilde{\gamma}_{1}(s)-\tilde{\gamma}_{1}\left(\eta_{2,1}\right)}{s+\eta_{1,1}}-\frac{\tilde{\gamma}_{1}(s)-\tilde{\gamma}_{1}\left(\eta_{2,1}\right)}{s-\eta_{2,1}}\right) \\
& +\frac{2 \lambda_{1} \theta \omega_{1,1} \omega_{2,1}}{\left(2 \lambda_{1}+\delta\right)\left(\omega_{1,1}+\omega_{2,1}\right)}\left(\frac{\tilde{\gamma}_{2}(s)-\tilde{\gamma}_{2}\left(\omega_{2,1}\right)}{s+\omega_{1,1}}-\frac{\tilde{\gamma}_{2}(s)-\tilde{\gamma}_{2}\left(\omega_{2,1}\right)}{s-\omega_{2,1}}\right) \\
& =\frac{\lambda_{1} \eta_{1,1} \eta_{2,1}}{\left(\lambda_{1}+\delta\right)\left(s+\eta_{1,1}\right)\left(s-\eta_{2,1}\right)}\left(\tilde{\gamma}_{1}\left(\eta_{2,1}\right)-\tilde{\gamma}_{1}(s)\right) \\
& +\frac{2 \lambda_{1} \theta \omega_{1,1} \omega_{2,1}}{\left(2 \lambda_{1}+\delta\right)\left(s+\omega_{1,1}\right)\left(s-\omega_{2,1}\right)}\left(\tilde{\gamma}_{2}\left(\omega_{2,1}\right)-\tilde{\gamma}_{2}(s)\right) \\
& =\frac{\lambda_{1} \eta_{1,1} \eta_{2,1}}{\left(\lambda_{1}+\delta\right)\left(s+\eta_{1,1}\right)\left(s-\eta_{2,1}\right)}\left(\tilde{\xi}_{1}\left(\eta_{2,1}\right) \tilde{\phi}_{1, w}\left(\eta_{2,1}\right)+\tilde{\chi}_{1}\left(\eta_{2,1}\right) \tilde{\phi}_{2, w}\left(\eta_{2,1}\right)\right. \\
& \left.+\tilde{\varphi}_{f-\theta h}\left(\eta_{2,1}\right)-\tilde{\xi}_{1}(s) \tilde{\phi}_{1, w}(s)-\tilde{\chi}_{1}(s) \tilde{\phi}_{2, w}(s)-\tilde{\varphi}_{f-\theta h}(s)\right) \\
& +\frac{2 \lambda_{1} \theta \omega_{1,1} \omega_{2,1}}{\left(2 \lambda_{1}+\delta\right)\left(s+\omega_{1,1}\right)\left(s-\omega_{2,1}\right)}\left(\tilde{\xi}_{2}\left(\omega_{2,1}\right) \tilde{\phi}_{1, w}\left(\omega_{2,1}\right)+\tilde{\chi}_{2}\left(\omega_{2,1}\right) \tilde{\phi}_{2, w}\left(\omega_{2,1}\right)\right. \\
& \left.+\tilde{\varphi}_{h}\left(\omega_{2,1}\right)-\tilde{\xi}_{2}(s) \tilde{\phi}_{1, w}(s)-\tilde{\chi}_{2}(s) \tilde{\phi}_{2, w}(s)-\tilde{\varphi}_{h}(s)\right) \\
& \tilde{\phi}_{2, w}(s)=\frac{\lambda_{2} \eta_{1,2} \eta_{2,2}}{\left(\lambda_{2}+\delta\right)\left(\eta_{1,2}+\eta_{2,2}\right)}\left(\tilde{m}_{2,1}(s)+T_{s} T_{\eta_{2,2}} \gamma_{1}(0)-\frac{1}{s+\eta_{1,2}} \tilde{\gamma}_{1}\left(\eta_{2,2}\right)\right) \\
& +\frac{2 \lambda_{2} \theta \omega_{1,2} \omega_{2,2}}{\left(2 \lambda_{2}+\delta\right)\left(\omega_{1,2}+\omega_{2,2}\right)}\left(\tilde{m}_{2,2}(s)+T_{s} T_{\omega_{2,2}} \gamma_{2}(0)-\frac{1}{s+\omega_{1,2}} \tilde{\gamma}_{2}\left(\omega_{2,2}\right)\right) \\
& =\frac{\lambda_{2} \eta_{1,2} \eta_{2,2}}{\left(\lambda_{2}+\delta\right)\left(\eta_{1,2}+\eta_{2,2}\right)}\left(\frac{\tilde{\gamma}_{1}(s)-\tilde{\gamma}_{1}\left(\eta_{2,2}\right)}{s+\eta_{1,2}}-\frac{\tilde{\gamma}_{1}(s)-\tilde{\gamma}_{1}\left(\eta_{2,2}\right)}{s-\eta_{2,2}}\right) \\
& +\frac{2 \lambda_{2} \theta \omega_{1,2} \omega_{2,2}}{\left(2 \lambda_{2}+\delta\right)\left(\omega_{1,2}+\omega_{2,2}\right)}\left(\frac{\tilde{\gamma}_{2}(s)-\tilde{\gamma}_{2}\left(\omega_{2,2}\right)}{s+\omega_{1,2}}-\frac{\tilde{\gamma}_{2}\left(\omega_{2,2}\right)-\tilde{\gamma}_{2}\left(\omega_{2,2}\right)}{s-\omega_{2,2}}\right) \\
& =\frac{\lambda_{2} \eta_{1,2} \eta_{2,2}}{\left(\lambda_{2}+\delta\right)\left(s+\eta_{1,2}\right)\left(s-\eta_{2,2}\right)}\left(\tilde{\gamma}_{1}\left(\eta_{2,2}\right)-\tilde{\gamma}_{1}(s)\right)
\end{aligned}
$$




$$
\begin{aligned}
& +\frac{2 \lambda_{2} \theta \omega_{1,2} \omega_{2,2}}{\left(2 \lambda_{2}+\delta\right)\left(s+\omega_{1,2}\right)\left(s-\omega_{2,2}\right)}\left(\tilde{\gamma}_{2}\left(\omega_{2,2}\right)-\tilde{\gamma}_{2}(s)\right) \\
= & \frac{\lambda_{1} \eta_{1,1} \eta_{2,1}}{\left(\lambda_{1}+\delta\right)\left(s+\eta_{1,1}\right)\left(s-\eta_{2,1}\right)}\left(\tilde{\xi}_{1}\left(\eta_{2,2}\right) \tilde{\phi}_{1, w}\left(\eta_{2,2}\right)+\tilde{\chi}_{1}\left(\eta_{2,2}\right) \tilde{\phi}_{2, w}\left(\eta_{2,2}\right)\right. \\
& \left.+\tilde{\varphi}_{f-\theta h}\left(\eta_{2,2}\right)-\tilde{\xi}_{1}(s) \tilde{\phi}_{1, w}(s)-\tilde{\chi}_{1}(s) \tilde{\phi}_{2, w}(s)-\tilde{\varphi}_{f-\theta h}(s)\right) \\
& +\frac{2 \lambda_{1} \theta \omega_{1,1} \omega_{2,1}}{\left(2 \lambda_{1}+\delta\right)\left(s+\omega_{1,1}\right)\left(s-\omega_{2,1}\right)}\left(\tilde{\xi}_{2}\left(\omega_{2,2}\right) \tilde{\phi}_{1, w}\left(\omega_{2,2}\right)+\tilde{\chi}_{2}\left(\omega_{2,2}\right) \tilde{\phi}_{2, w}\left(\omega_{2,2}\right)\right. \\
& \left.+\tilde{\varphi}_{h}\left(\omega_{2,2}\right)-\tilde{\xi}_{2}(s) \tilde{\phi}_{1, w}(s)-\tilde{\chi}_{2}(s) \tilde{\phi}_{2, w}(s)-\tilde{\varphi}_{h}(s)\right)
\end{aligned}
$$

And

$$
\begin{gathered}
\tilde{\phi}_{1, w}\left(\eta_{2,1}\right)=\frac{2 \lambda_{1} \theta \omega_{1,1} \omega_{2,1}}{\left(2 \lambda_{1}+\delta\right)\left(\eta_{2,1}+\omega_{1,1}\right)\left(\eta_{2,1}-\omega_{2,1}\right)}\left(\tilde{\gamma}_{2}\left(\omega_{2,1}\right)-\tilde{\gamma}_{2}\left(\eta_{2,1}\right)\right) . \\
\tilde{\phi}_{1, w}\left(\omega_{2,1}\right)=\frac{\lambda_{1} \eta_{1,1} \eta_{2,1}}{\left(\lambda_{1}+\delta\right)\left(\omega_{2,1}+\eta_{1,1}\right)\left(\omega_{2,1}-\eta_{2,1}\right)}\left(\tilde{\gamma}_{1}\left(\eta_{2,1}\right)-\tilde{\gamma}_{1}\left(\omega_{2,1}\right)\right) . \\
\tilde{\phi}_{2, w}\left(\eta_{2,2}\right)=\frac{2 \lambda_{2} \theta \omega_{1,2} \omega_{2,2}}{\left(2 \lambda_{2}+\delta\right)\left(\eta_{2,2}+\omega_{1,2}\right)\left(\eta_{2,2}-\omega_{2,2}\right)}\left(\tilde{\gamma}_{2}\left(\omega_{2,2}\right)-\tilde{\gamma}_{2}\left(\eta_{2,2}\right)\right) . \\
\tilde{\phi}_{2, w}\left(\omega_{2,2}\right)=\frac{\lambda_{2} \eta_{1,2} \eta_{2,2}}{\left(\lambda_{2}+\delta\right)\left(\omega_{2,2}+\eta_{1,2}\right)\left(s-\eta_{2,2}\right)}\left(\tilde{\gamma}_{1}\left(\eta_{2,2}\right)-\tilde{\gamma}_{1}\left(\omega_{2,2}\right)\right) .
\end{gathered}
$$

Theorem 3. The Laplace transforms of the Gerber-Shiu functions given (4.8) and (4.9) are

$$
\begin{aligned}
&\left(1+A_{1} \tilde{\xi}_{1}(s)+B_{1} \tilde{\xi}_{2}(s)\right) \tilde{\phi}_{1, w}(s)+\left(A_{1} \tilde{\chi}_{1}(s)+B_{1} \tilde{\chi}_{2}(s)\right) \tilde{\phi}_{2, w}(s) \\
&= A_{1}\left(\tilde{\xi}_{1}\left(\eta_{2,1}\right) \tilde{\phi}_{1, w}\left(\eta_{2,1}\right)+\tilde{\chi}_{1}\left(\eta_{2,1}\right) \tilde{\phi}_{2, w}\left(\eta_{2,1}\right)+\tilde{\varphi}_{f-\theta h}\left(\eta_{2,1}\right)-\tilde{\varphi}_{f-\theta h}(s)\right) \\
&+B_{1}\left(\tilde{\xi}_{2}\left(\omega_{2,1}\right) \tilde{\phi}_{1, w}\left(\omega_{2,1}\right)+\tilde{\chi}_{2}\left(\omega_{2,1}\right) \tilde{\phi}_{2, w}\left(\omega_{2,1}\right)+\tilde{\varphi}_{h}\left(\omega_{2,1}\right)-\tilde{\varphi}_{h}(s)\right) \\
&\left.\left(1+A_{2} \tilde{\xi}_{1}(s)+B_{2} \tilde{\xi}_{2}(s)\right) \tilde{\phi}_{1, w}(s)+\left(A_{2} \tilde{\chi}_{1}(s)+B_{2} \tilde{\chi}_{2}(s)\right) \tilde{\phi}_{2, w}(s)-\tilde{\varphi}_{f-\theta h}(s)\right) A_{2}\left(\tilde{\xi}_{1}\left(\eta_{2,2}\right) \tilde{\phi}_{1, w}\left(\eta_{2,2}\right)+\tilde{\chi}_{1}\left(\eta_{2,2}\right) \tilde{\phi}_{2, w}\left(\eta_{2,2}\right)+\tilde{\varphi}_{f-\theta h}\left(\eta_{2,2}\right)\right) \\
&+ B_{2}\left(\tilde{\xi}_{2}\left(\omega_{2,2}\right) \tilde{\phi}_{1, w}\left(\omega_{2,2}\right)+\tilde{\chi}_{2}\left(\omega_{2,2}\right) \tilde{\phi}_{2, w}\left(\omega_{2,2}\right)+\tilde{\varphi}_{h}\left(\omega_{2,2}\right)-\tilde{\varphi}_{h}(s)\right) \\
& A_{1}=\frac{\lambda_{1} \eta_{1,1} \eta_{2,1}}{\left(\lambda_{1}+\delta\right)\left(s+\eta_{1,1}\right)\left(s-\eta_{2,1}\right)}, \quad B_{1}=\frac{2 \lambda_{1} \theta \omega_{1,1} \omega_{2,1}}{\left(2 \lambda_{1}+\delta\right)\left(s+\omega_{1,1}\right)\left(s-\omega_{2,1}\right)}, \\
& A_{2}=\frac{\lambda_{2} \eta_{1,2} \eta_{2,2}}{\left(\lambda_{2}+\delta\right)\left(s+\eta_{1,2}\right)\left(s-\eta_{2,2}\right)}, \quad B_{2}=\frac{2 \lambda_{1} \theta \omega_{1,2} \omega_{2,2}}{\left(2 \lambda_{1}+\delta\right)\left(s+\omega_{1,2}\right)\left(s-\omega_{2,2}\right)} .
\end{aligned}
$$

And then, we consider the Laplace transforms of $\tilde{n}_{1, i}(s)$

$$
\begin{gathered}
\tilde{n}_{1,1}(s)=\int_{0}^{\infty} \mathrm{e}^{-s y} \int_{0}^{y} \mathrm{e}^{-\eta_{1,1}(y-v)} \alpha_{1}(v) \mathrm{d} v \mathrm{~d} y=\frac{1}{s+\eta_{1,1}} \tilde{\alpha}_{1}(s) \\
\int_{0}^{\infty} \mathrm{e}^{-s u} \mathrm{e}^{-\eta_{1,1} u} \tilde{\alpha}_{1}\left(\eta_{2,1}\right) \mathrm{d} u=\tilde{\alpha}_{1}\left(\eta_{2,1}\right) \int_{0}^{\infty} \mathrm{e}^{-\left(s+\eta_{1,1}\right) u} \mathrm{~d} u=\frac{1}{s+\eta_{1,1}} \tilde{\alpha}_{1}\left(\eta_{2,1}\right) \\
\tilde{n}_{1,2}(s)=\int_{0}^{\infty} \mathrm{e}^{-s y} \int_{0}^{y} \mathrm{e}^{-\omega_{1,1}(y-v)} \alpha_{2}(v) \mathrm{d} v=\frac{1}{s+\omega_{1,1}} \tilde{\alpha}_{2}(s)
\end{gathered}
$$




$$
\begin{gathered}
\int_{0}^{\infty} \mathrm{e}^{-s u} \mathrm{e}^{-\omega_{1,1} u} \tilde{\alpha}_{2}\left(\omega_{2,1}\right) \mathrm{d} u=\tilde{\alpha}_{2}\left(\omega_{2,1}\right) \int_{0}^{\infty} \mathrm{e}^{-\left(s+\omega_{1,1}\right) u} \mathrm{~d} u=\frac{1}{s+\omega_{1,1}} \tilde{\alpha}_{2}\left(\omega_{2,1}\right) \\
\tilde{n}_{2,1}(s)=\int_{0}^{\infty} \mathrm{e}^{-s y} \int_{0}^{y} \mathrm{e}^{-\eta_{1,2}(y-v)} \alpha_{1}(v) \mathrm{d} v \mathrm{~d} y=\frac{1}{s+\eta_{1,2}} \tilde{\alpha}_{1}(s) \\
\int_{0}^{\infty} \mathrm{e}^{-s u} \mathrm{e}^{-\eta_{1,2} u} \tilde{\alpha}_{1}\left(\eta_{2,2}\right) \mathrm{d} u=\tilde{\alpha}_{1}\left(\eta_{2,2}\right) \int_{0}^{\infty} \mathrm{e}^{-\left(s+\eta_{1,2}\right) u} \mathrm{~d} u=\frac{1}{s+\eta_{1,2}} \tilde{\alpha}_{1}\left(\eta_{2,2}\right) \\
\tilde{n}_{2,2}(s)=\int_{0}^{\infty} \mathrm{e}^{-s y} \int_{0}^{y} \mathrm{e}^{-\omega_{1,2}(y-v)} \alpha_{2}(v) \mathrm{d} v=\frac{1}{s+\omega_{1,2}} \tilde{\alpha}_{2}(s) \\
\int_{0}^{\infty} \mathrm{e}^{-s u} \mathrm{e}^{-\omega_{1,2} u} \tilde{\alpha}_{2}\left(\omega_{2,2}\right) \mathrm{d} u=\tilde{\alpha}_{2}\left(\omega_{2,2}\right) \int_{0}^{\infty} \mathrm{e}^{-\left(s+\omega_{1,2}\right) u} \mathrm{~d} u=\frac{1}{s+\omega_{1,2}} \tilde{\alpha}_{2}\left(\omega_{2,2}\right)(5.2
\end{gathered}
$$

So, we could get the Laplace transport

$$
\begin{aligned}
& \tilde{\phi}_{1, d}(s)=\frac{1}{s+\eta_{1,1}}+\frac{\lambda_{1} \eta_{1,1} \eta_{2,1}}{\left(\lambda_{1}+\delta\right)\left(\eta_{1,1}+\eta_{2,1}\right)} \\
& \times\left[\frac{1}{s+\eta_{1,1}} \tilde{\alpha}_{1}(s)+T_{s} T_{\eta_{2,1}} \alpha_{1}(0)-\frac{1}{s+\eta_{1,1}} \tilde{\alpha}_{1}\left(\eta_{2,1}\right)\right] \\
& +\frac{2 \lambda_{1} \theta \omega_{1,1} \omega_{2,1}}{\left(2 \lambda_{1}+\delta\right)\left(\omega_{1,1}+\omega_{2,1}\right)}\left[\frac{1}{s+\omega_{1,1}} \tilde{\alpha}_{2}(s)+T_{s} T_{\omega_{2,1}} \alpha_{2}(0)-\frac{1}{s+\omega_{1,1}} \tilde{\alpha}_{2}\left(\omega_{2,1}\right)\right] \\
& =\frac{1}{s+\eta_{1,1}}+\frac{\lambda_{1} \eta_{1,1} \eta_{2,1}}{\left(\lambda_{1}+\delta\right)\left(s+\eta_{1,1}\right)\left(s-\eta_{2,1}\right)}\left(\tilde{\alpha}_{1}\left(\eta_{2,1}\right)-\tilde{\alpha}_{1}(s)\right) \\
& +\frac{2 \lambda_{1} \theta \omega_{1,1} \omega_{2,1}}{\left(2 \lambda_{1}+\delta\right)\left(s+\omega_{1,1}\right)\left(s-\omega_{2,1}\right)}\left(\tilde{\alpha}_{2}\left(\omega_{2,1}\right)-\tilde{\alpha}_{2}(s)\right) \\
& =\frac{1}{s+\eta_{1,1}}+\frac{\lambda_{1} \eta_{1,1} \eta_{2,1}}{\left(\lambda_{1}+\delta\right)\left(s+\eta_{1,1}\right)\left(s-\eta_{2,1}\right)} \\
& \times\left(\tilde{\xi}_{1}\left(\eta_{2,1}\right) \tilde{\phi}_{1, d}\left(\eta_{2,1}\right)+\tilde{\chi}_{1}\left(\eta_{2,1}\right) \tilde{\phi}_{2, d}\left(\eta_{2,1}\right)-\tilde{\xi}_{1}(s) \tilde{\phi}_{1, d}(s)-\tilde{\chi}_{1}(s) \tilde{\phi}_{2, d}(s)\right) \\
& +\frac{2 \lambda_{1} \theta \omega_{1,1} \omega_{2,1}}{\left(2 \lambda_{1}+\delta\right)\left(s+\omega_{1,1}\right)\left(s-\omega_{2,1}\right)} \\
& \times\left(\tilde{\xi}_{2}\left(\omega_{2,1}\right) \tilde{\phi}_{1, d}\left(\omega_{2,1}\right)+\tilde{\chi}_{2}\left(\omega_{2,1}\right) \tilde{\phi}_{2, d}\left(\omega_{2,1}\right)-\tilde{\xi}_{2}(s) \tilde{\phi}_{1, d}(s)-\tilde{\chi}_{2}(s) \tilde{\phi}_{2, d}(s)\right) \\
& \tilde{\phi}_{2, d}(s)=\frac{1}{s+\eta_{1,2}}+\frac{\lambda_{2} \eta_{1,2} \eta_{2,2}}{\left(\lambda_{2}+\delta\right)\left(s+\eta_{1,2}\right)\left(s-\eta_{2,2}\right)} \\
& \times\left(\tilde{\xi}_{1}\left(\eta_{2,2}\right) \tilde{\phi}_{1, d}\left(\eta_{2,2}\right)+\tilde{\chi}_{1}\left(\eta_{2,2}\right) \tilde{\phi}_{2, d}\left(\eta_{2,2}\right)-\tilde{\xi}_{1}(s) \tilde{\phi}_{1, d}(s)-\tilde{\chi}_{1}(s) \tilde{\phi}_{2, d}(s)\right) \\
& +\frac{2 \lambda_{1} \theta \omega_{1,2} \omega_{2,2}}{\left(2 \lambda_{1}+\delta\right)\left(s+\omega_{1,2}\right)\left(s-\omega_{2,2}\right)} \\
& \times\left(\tilde{\xi}_{2}\left(\omega_{2,2}\right) \tilde{\phi}_{1, d}\left(\omega_{2,2}\right)+\tilde{\chi}_{2}\left(\omega_{2,2}\right) \tilde{\phi}_{2, d}\left(\omega_{2,2}\right)-\tilde{\xi}_{2}(s) \tilde{\phi}_{1, d}(s)-\tilde{\chi}_{2}(s) \tilde{\phi}_{2, d}(s)\right)
\end{aligned}
$$

Theorem 4. The Laplace transforms of the Gerber-Shiu functions given (4.19) and (4.20) are

$$
\begin{aligned}
& \left(1+A_{1} \tilde{\xi}_{1}(s)+B_{1} \tilde{\xi}_{2}(s)\right) \tilde{\phi}_{1, d}(s)+\left(A_{1} \tilde{\chi}_{1}(s)+B_{1} \tilde{\chi}_{1}(s)\right) \tilde{\phi}_{2, d}(s) \\
& =\frac{1}{s+\eta_{1,1}}+A_{1}\left(\tilde{\xi}_{1}\left(\eta_{2,1}\right) \tilde{\phi}_{1, d}\left(\eta_{2,1}\right)+\tilde{\chi}_{1}\left(\eta_{2,1}\right) \tilde{\phi}_{2, d}\left(\eta_{2,1}\right)\right) \\
& \quad+B_{2}\left(\tilde{\xi}_{2}\left(\omega_{2,1}\right) \tilde{\phi}_{1, d}\left(\omega_{2,1}\right)+\tilde{\chi}_{2}\left(\omega_{2,1}\right) \tilde{\phi}_{2, d}\left(\omega_{2,1}\right)\right)
\end{aligned}
$$




$$
\begin{aligned}
& \left(A_{2} \tilde{\xi}_{1}(s)+B_{2} \tilde{\xi}_{2}(s)\right) \tilde{\phi}_{1, d}(s)+\left(1+A_{2} \tilde{\chi}_{1}(s)+B_{2} \tilde{\chi}_{2}(s)\right) \tilde{\phi}_{2, d}(s) \\
= & \frac{1}{s+\eta_{1,2}}+A_{2}\left(\tilde{\xi}_{1}\left(\eta_{2,2}\right) \tilde{\phi}_{1, d}\left(\eta_{2,2}\right)+\tilde{\chi}_{1}\left(\eta_{2,2}\right) \tilde{\phi}_{2, d}\left(\eta_{2,2}\right)\right) \\
& +B_{2}\left(\tilde{\xi}_{2}\left(\omega_{2,2}\right) \tilde{\phi}_{1, d}\left(\omega_{2,2}\right)+\tilde{\chi}_{2}\left(\omega_{2,2}\right) \tilde{\phi}_{2, d}\left(\omega_{2,2}\right)\right) \\
A_{1}= & \frac{\lambda_{1} \eta_{1,1} \eta_{2,1}}{\left(\lambda_{1}+\delta\right)\left(s+\eta_{1,1}\right)\left(s-\eta_{2,1}\right)}, \quad B_{1}=\frac{2 \lambda_{1} \theta \omega_{1,1} \omega_{2,1}}{\left(2 \lambda_{1}+\delta\right)\left(s+\omega_{1,1}\right)\left(s-\omega_{2,1}\right)}, \\
A_{2}= & \frac{\lambda_{2} \eta_{1,2} \eta_{2,2}}{\left(\lambda_{2}+\delta\right)\left(s+\eta_{1,2}\right)\left(s-\eta_{2,2}\right)}, \quad B_{2}=\frac{2 \lambda_{1} \theta \omega_{1,2} \omega_{2,2}}{\left(2 \lambda_{1}+\delta\right)\left(s+\omega_{1,2}\right)\left(s-\omega_{2,2}\right)} .
\end{aligned}
$$

\section{Conclusion Remarks}

Researchers are more and more inclined to study the theory of ruin in combination with the actual situation. But the actual situations are various, and they could not be show out just in one way. We will continue to study the dependent risk model and get the solution of the Laplace transform of the integro-differential equation in the dependent risk model. We can consider more risk models by changing the expressions of disturbance and premium rate. The disturbance could be a levy process or a jump process and so on, meanwhile the premium rate could be linear function. There are many follow-up studies that need to be done.

\section{Acknowledgements}

The research was supported by the National Natural Science Foundation of Shandong (No. ZR2015AL009).

\section{Conflicts of Interest}

The author declares no conflicts of interest regarding the publication of this paper.

\section{References}

[1] Tsai, C.L. (2003) On the Expectations of the Present Values of the Time of Ruin Perturbed by Diffusion. Insurance: Mathematics and Economics, 32, 413-429. https://doi.org/10.1016/S0167-6687(03)00130-6

[2] Tsai, C.L. and Willmot, G.E. (2002) A Generalized Defective Renewal Equation for the Surplus Process Perturbed by Diffusion. Insurance: Mathematics and Economics, 30, 51-66. https://doi.org/10.1016/S0167-6687(01)00096-8

[3] Tsai, C.L. and Willmot, G.E. (2002) On the Moments of the Surplus Process Perturbed by Diffusion. Insurance: Mathematics and Economics, 31, 327-350. https://doi.org/10.1016/S0167-6687(02)00159-2

[4] Gerber, H.U. and Shiu, E.S.W. (1998) On the Time Value of Ruin. North American Actuarial Journal, 2, 48-72. https://doi.org/10.1080/10920277.1998.10595671

[5] Gerber, H.U. and Shiu, E.S. (2005) The Time Value of Ruin in a Sparre Andersen Model. North American Actuarial Journal, 9, 49-69. https://doi.org/10.1080/10920277.2005.10596197 
[6] Albrecher, H. and Boxma, O.J. (2004) A Ruin Model with Dependence between Claim Sizes and Claim Intervals. Insurance: Mathematics and Economics, 35, 245-254. https://doi.org/10.1016/j.insmatheco.2003.09.009

[7] Albrecher, H. and Teugels, J.L. (2006) Exponential Behavior in the Presence of Dependence in Risk Theory. Journal of Applied Probability, 43, 257-273. https://doi.org/10.1239/jap/1143936258

[8] Carbno, C. (2007) Actuarial Theory for Dependent Risks: Measures, Orders, and Models. Technometrics, 49, 495. https://doi.org/10.1198/tech.2007.s690

[9] Cossette, H. and Marri, E.M.F. (2010) Analysis of Ruin Measures for the Classical Compound Poisson Risk Model with Dependence. Scandinavian Actuarial Journal, 2010, 221-245. https://doi.org/10.1080/03461230903211992

[10] Dufresne, F. and Gerber, H.U. (1991) Risk Theory for the Compound Poisson Process That Is Perturbed by Diffusion. Insurance: Mathematics and Economics, 10, 51-59. https://doi.org/10.1016/0167-6687(91)90023-Q

[11] Boudreault, M., Cossette, H., Landriault, D. and Marceau, E. (2006) On a Risk Model with Dependence between Interclaim Arrivals and Claim Sizes. Scandinavian Actuarial Journal, 2006, 301-323. https://doi.org/10.1080/03461230600992266

[12] Meng, Q., Zhang, X. and Guo, J. (2008) On a Risk Model with Dependence between Claim Sizes and Claim Intervals. Statistics and Probability Letters, 78, 1727-1734. https://doi.org/10.1016/j.spl.2008.01.031

[13] Zhang, Z. and Yang, H. (2011) Gerber-Shiu Analysis in a Perturbed Risk Model with Dependence between Claim Sizes and Interclaim Times. Journal of Computational and Applied Mathematics, 235, 1189-1204. https://doi.org/10.1016/j.cam.2010.08.003

[14] Li, Z. and Sendova, K.P. (2015) On a Ruin Model with Both Interclaim Times and Premiums Depending on Claim Sizes. Scandinavian Actuarial Journal, 2015, 245-265. https://doi.org/10.1080/03461238.2013.811096

[15] Zhou, M. and Cai, J. (2009) A Perturbed Risk Model with Dependence between Premium Rates and Claim Sizes. Insurance: Mathematics and Economics, 45, 382-392. https://doi.org/10.1016/j.insmatheco.2009.08.008

[16] Borodin, A.N. and Salminen, P. (2002) Handbook of Brownian Motion-Facts and Formulae (Probability and Its Applications). Birkhäuser, Basel, 657-658. https://doi.org/10.1007/978-3-0348-7652-0 